\title{
Nonlinear dynamics of double-layer unstable interfaces with non-uniform velocity shear
}

\section{Chihiro Matsuoka}

\begin{tabular}{|c|l|}
\hline Citation & Physics of Fluids. 32(10); 102109. \\
\hline Issue Date & $2020-10-16$ \\
\hline Type & Journal Article \\
\hline Textversion & Publisher \\
\hline Rights & $\begin{array}{l}\text { This article may be downloaded for personal use only. Any other use requires prior } \\
\text { permission of the author and AIP Publishing. This article appeared in Physics of Fields, } \\
\text { Volume 32, Issue 10, and may be found at https://doi.org/10.1063/5.0023558. }\end{array}$ \\
\hline DOI & $10.1063 / 5.0023558$ \\
\hline
\end{tabular}

\section{Self-Archiving by Author(s)}

Placed on: Osaka City University Repository 


\title{
Nonlinear dynamics of double-layer unstable interfaces with non-uniform velocity shear
}

\author{
Cite as: Phys. Fluids 32, 102109 (2020); doi: 10.1063/5.0023558 \\ Submitted: 30 July 2020 - Accepted: 30 September 2020 • \\ Published Online: 16 October 2020
}

Chihiro Matsuoka (松岡千博) ${ }^{a)}$

\begin{abstract}
AFFILIATIONS
Laboratory of Applied Mathematics, Graduate School of Engineering, Osaka City University, Sugimoto, Sumiyoshi, Osaka 558-8585, Japan
\end{abstract}

\begin{abstract}
a) Author to whom correspondence should be addressed: cmatsuoka@osaka-cu.ac.jp. Also at: Nambu Yoichiro Institute of Theoretical and Experimental Physics (NITEP), Osaka City University, Osaka City University Advanced Mathematical Institute (OCAMI), Osaka, Japan.
\end{abstract}

\begin{abstract}
The interaction of double-layer density stratified interfaces with initial non-uniform velocity shear is investigated theoretically and numerically, taking the incompressible Richtmyer-Meshkov instability as an example. The linear analysis for providing the initial conditions in numerical calculations is performed, and some numerical examples of vortex double layers are presented using the vortex sheet model. We show that the density stratifications (Atwood numbers), the initial distance between two interfaces, and the distribution of the initial velocity shear determine the evolution of vortex double layers. When the Atwood numbers are large, the deformation of interfaces is small, and the distance between the two interfaces is almost unchanged. On the other hand, when the Atwood numbers are small and the initial distance between two interfaces is sufficiently close (less than or equal to the half of the wavelength of the initial disturbance), the two interfaces get closer to each other and merge at the last computed stage due to the incompressibility. We confirm this theoretically expected fact numerically.
\end{abstract}

Published under license by AIP Publishing. https://doi.org/10.1063/5.0023558

\section{INTRODUCTION}

The motion of unstable interfaces in multi-layer flow is important in various areas such as internal gravity waves, ${ }^{1,2}$ geophysical fluid dynamics, ${ }^{3}$ and plasma physics. ${ }^{4-8}$ Karman vortex streets that appear in the wake region ${ }^{9,10}$ are also the significant examples of unstable double-layer interfaces. These multi-layer interfaces often occur in the fluids with density stratification, and they interact with each other when the interfaces are located close together. The Rayleigh-Taylor instability (RTI) ${ }^{4,5}$ and the Richtmyer-Meshkov instability $(\mathrm{RMI})^{7,11,12}$ are the examples of such density stratification instabilities.

RMI is a shock-induced interfacial instability that occurs at a corrugated interface separating two fluids with density jump..$^{11,12}$ This instability plays an important role in various fields such as astrophysical supernova explosion, ${ }^{13-17}$ supersonic combustion, inertial confinement fusion (ICF), ${ }^{18-20}$ and fundamental plasma physics. $^{21,22}$ In the linear stage of RMI immediately after the passage of shocks, the compressibility effects by the shocks are dominant, ${ }^{23-31}$ and the amplitude of the interface is sufficiently small. When the shocks leave the interface (more than one wavelength), the system can be regarded as incompressible and irrotational except at the interface, on which non-uniform vorticity is induced by the shocks. ${ }^{21,32}$ In this incompressible nonlinear stage, it is known that the interface of RMI rolls up like a mushroom, ${ }^{32,33}$ and we can treat that interface as a non-uniform vortex sheet. ${ }^{34-36}$ For the incompressible model of RMI, various analytical studies, such as weakly nonlinear analysis ${ }^{32,37,38}$ or the asymptotic growth velocity and curvature of bubbles, have also been provided. ${ }^{39}$

In real laboratory experiments, the density jump in RMI in the interface is not necessarily sharp, and the system is often composed of multi-layer interfaces with density stratifications. Mikaelian theoretically investigated such multi-layer systems ${ }^{4-7}$ and derived the eigenvalues and eigenfunctions in the multi-component density stratified fluids. His linear analysis is applicable not only to multi-component RTI and RMI but also to multi-layer internal gravity waves. ${ }^{1}$ Extending the linear theory for the $M(M=2,3, \ldots)$ 
component density stratified fluids by Mikaelian, Liu et al. ${ }^{8}$ performed a weakly nonlinear analysis for the incompressible RMI with three-component fluids in which two interfaces exist. In that study, they concluded that the distance between two interfaces largely affects the growth of amplitudes of the interfaces. In order to obtain the finite thickness effect on harmonics in RMI, they assume that both sides of the two interfaces are vacuum.

In addition to the above linear and weakly nonlinear analyses for multi-component density stratified systems, there also exists a numerical study that investigated the interaction of vortex double layers in RMI. Zabusky and Zhang ${ }^{41}$ investigated the shock inclinedcurtain interaction of a planar RMI using the piecewise parabolic method (PPM) algorithm. ${ }^{42}$ In this study, they found the appearance and interaction of opposite-signed vortex double layers and the emergence of dipolar vortex domains. The two vortex layers (interfaces) approach each other and form a complicated vortical structure. In their study, ${ }^{41}$ the authors insist that the vortex double layer is fundamental in understanding the turbulent mixing inherent in accelerated inhomogeneous flows and the reacceleration problem such as the reshock RMI. ${ }^{43}$ This is the first work showing that the finite-amplitude double-layer solution can exist in RMI. However, the initial conditions or parameter dependence, such as the Atwood numbers and the distance between two interfaces, was unknown in their numerical study.

Despite its importance, there does not exist the theoretical work that treated the nonlinear behavior of the multi-layer interfaces with density stratification. In the current study, we first perform the linear analysis for the double-layer RMI and determine the initial conditions for numerical calculations. After that, we present the theoretical model for solving the nonlinear interaction of double-layer interfaces in three-component RMI and investigate the long-time behavior of that using the boundary integral method $^{34,35,44-47}$ and the vortex (blob) method. ${ }^{34-36,48-52}$ These two methods are usually distinguished by the fact whether the parameter $\delta^{49,50}$ to regularize the vortex induced velocity (Birkhoff-Rott equation $)^{53-55}$ is zero (the boundary integral method) or finite values (the vortex method), but the thickness of the interface is assumed to be zero in both methods. From now on, we refer to both of these methods as the vortex sheet model (VSM). VSM can capture the long-time nonlinear evolution of two-dimensional shear flows when the computation is regularized. ${ }^{49}$ It is known that the regularized vortex motion is not an exact solution to the Euler equation; ${ }^{56}$ however, the model equation effectively describes real interfacial motion such as roll-ups ${ }^{49,57}$ together with the velocity field in bulk. ${ }^{17,58,59}$

Using the VSM, we regard the double-layer interfacial motion in RMI as the motion of two vortex sheets. In the VSM, the interfacial velocity is described by a Lagrangian parameter that parameterizes the interface. In the current study, we parameterize two interfaces by the same Lagrangian parameter. When two interfaces exist in the system, we have two interfacial velocities, which correspond to the fact that two Lagrange derivatives with respect to time exist. Extending the case of one interface, we define the two interfacial velocities as the deviation from the average fluid velocity (refer to Sec. II) so that we can calculate the model equations with a Lagrangian time derivative.

Since we consider three-component RMI, two Atwood numbers exist in the system. We present various interfacial motions together with the growth velocities of bubbles and spikes by varying those Atwood numbers and initial conditions including the distance between the two interfaces. We show that the two interfaces gradually approach each other for all cases due to the incompressibility. The incompressibility indicates the area-preserving. Therefore, the initial area between the two interfaces should be conserved mathematically, no matter how large the interfacial deformation becomes. When the initial distance between the two interfaces is sufficiently small (less than or equal to the half of the wavelength of the initial perturbation), the two interfaces merge and behave like one vortex sheet at the last computed stage, although they do not coincide exactly to maintain the initial area between them. The merging phenomenon between two vortex sheets is also observed in the numerical simulations by Zabusky and Zhang. ${ }^{41}$ Although we select incompressible RMI as a physical example of multi-layer systems, the model and analysis developed here are applicable to general unstable multi-layer interfacial motions, such as the KelvinHelmholtz instability (KHI), RTI, and internal gravity waves. In the current study, we consider the three-component fluid systems; however, the extension of the model equations to $M(M \geq 3)$ component density stratified fluids is not difficult.

This paper is organized as follows. In Sec. II, we provide the mathematical model for the three-component density stratified fluid such that two interfaces coexist in the system and derive the governing equations for describing the motion of the interfaces as vortex sheets. In Sec. III, the linear analysis for deriving the initial conditions for numerical calculations is performed. In Sec. IV, we present some numerical results for the motion of the two vortex sheets in RMI varying the Atwood numbers and initial distances between the two interfaces. Section $V$ is devoted to conclusion and discussions.

\section{FORMULATION OF THE PROBLEM}

We consider two-dimensional inviscid and incompressible flows in which two fluid interfaces $I_{1}$ and $I_{2}$ with density and tangential velocity jumps (Fig. 1). The fluids over $I_{1}$ (density $\rho_{0}$ ) and

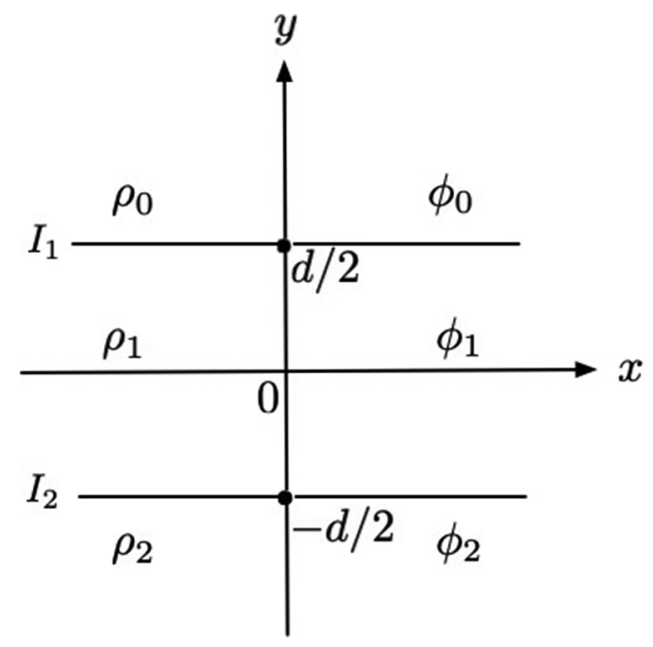

FIG. 1. Schematic figure of the physical situation. 
under $I_{2}$ (density $\rho_{2}$ ) are assumed to extend to infinity (Fig. 1). We also assume that the fluid is irrotational except the interfaces. Then, the Bernoulli equation holds in each fluid $i$,

$$
\rho_{i}\left[\frac{\partial \phi_{i}}{\partial t}+\frac{1}{2}\left(\nabla \phi_{i}\right)^{2}\right]+p_{i}=C_{i}(t) \quad(i=0,1,2)
$$

where $\phi_{i}(i=0,1,2)$ is the velocity potential that is related to the fluid velocity $\boldsymbol{v}_{i}$ as $\boldsymbol{v}_{i}=\nabla \phi_{i}, \rho_{i}$ is the density of fluid $i, p_{i}$ is the pressure, and $C_{i}(t)$ is some constant depending only on time. When we ignore the gravity, we can set $C_{0}(t)=C_{1}(t)=C_{2}(t)$ in Eq. (1). Since the system is described by the potential flow, the Laplace equation

$$
\triangle \phi_{i}=0 \quad(i=0,1,2)
$$

holds for the velocity potential $\phi_{i}$ in each fluid region across the interfaces.
From the pressure continuous condition $p_{i}=p_{i-1}$ at each interface $I_{i}(i=1,2)$, Eq. (1) yields

$$
\begin{aligned}
\left(1-A_{i}\right) & {\left[\frac{\partial \phi_{i}}{\partial t}+\frac{1}{2}\left(\nabla \phi_{i}\right)^{2}\right] } \\
& -\left(1+A_{i}\right)\left[\frac{\partial \phi_{i-1}}{\partial t}+\frac{1}{2}\left(\nabla \phi_{i-1}\right)^{2}\right]=0 \quad(i=1,2)
\end{aligned}
$$

where the Atwood number $A_{i}$, the density ratio between fluids $i$ and $i-1$, is defined by

$$
A_{i}=\frac{\rho_{i-1}-\rho_{i}}{\rho_{i-1}+\rho_{i}} \quad(i=1,2) .
$$

We parameterize points on the interface $\boldsymbol{x}=\boldsymbol{X}_{i}(i=1,2)$ as

$$
\boldsymbol{X}_{i}(e, t)=\left[X_{i}(e, t), Y_{i}(e, t)\right]
$$

using the same Lagrangian parameter $e(-\pi \leq e \leq \pi)$, where we assume the periodicity in the $x$ direction. Due to the linearity of the field (2), the vortex induced velocity $\boldsymbol{W}_{i}=\left(W_{i, x}, W_{i, y}\right)(i=1$, 2) on the interface $I_{i}$ is given as the sum of the self-contribution of interface $I_{i}$ and the contribution from the other interface $I_{j}(i \neq j)$ as follows:

$$
\begin{aligned}
W_{i, x}\left(X_{i}(e, t), Y_{i}(e, t)\right)= & -\frac{1}{4 \pi} \mathrm{P} . \mathrm{V} \cdot \int_{-\pi}^{\pi} \frac{\gamma_{i}\left(e^{\prime}, t\right) s_{i, e}\left(e^{\prime}, t\right) \sinh \left(Y_{i}(e, t)-Y_{i}\left(e^{\prime}, t\right)\right)}{\cosh \left(Y_{i}(e, t)-Y_{i}\left(e^{\prime}, t\right)\right)-\cos \left(X_{i}(e, t)-X_{i}\left(e^{\prime}, t\right)\right)+\delta^{2}} d e^{\prime} \\
& -\frac{1}{4 \pi} \int_{-\pi}^{\pi} \frac{\gamma_{j}\left(e^{\prime}, t\right) s_{j, e}\left(e^{\prime}, t\right) \sinh \left(Y_{i}(e, t)-Y_{j}\left(e^{\prime}, t\right)\right)}{\cosh \left(Y_{i}(e, t)-Y_{j}\left(e^{\prime}, t\right)\right)-\cos \left(X_{i}(e, t)-X_{j}\left(e^{\prime}, t\right)\right)+\delta^{2}} d e^{\prime} \quad(i \neq j), \\
W_{i, y}\left(X_{i}(e, t), Y_{i}(e, t)\right)= & \frac{1}{4 \pi} \mathrm{P} . \mathrm{V} . \int_{-\pi}^{\pi} \frac{\gamma_{i}\left(e^{\prime}, t\right) s_{i, e}\left(e^{\prime}, t\right) \sin \left(X_{i}(e, t)-X_{i}\left(e^{\prime}, t\right)\right)}{\cosh \left(Y_{i}(e, t)-Y_{i}\left(e^{\prime}, t\right)\right)-\cos \left(X_{i}(e, t)-X_{i}\left(e^{\prime}, t\right)\right)+\delta^{2}} d e^{\prime} \\
& +\frac{1}{4 \pi} \int_{-\pi}^{\pi} \frac{\gamma_{j}\left(e^{\prime}, t\right) s_{j, e}\left(e^{\prime}, t\right) \sin \left(X_{i}(e, t)-X_{j}\left(e^{\prime}, t\right)\right)}{\cosh \left(Y_{i}(e, t)-Y_{j}\left(e^{\prime}, t\right)\right)-\cos \left(X_{i}(e, t)-X_{j}\left(e^{\prime}, t\right)\right)+\delta^{2}} d e^{\prime} \quad(i \neq j),
\end{aligned}
$$

in which $\boldsymbol{\gamma}_{i}=\boldsymbol{u}_{i}-\boldsymbol{u}_{i-1}$, and $\gamma_{i}=\boldsymbol{\gamma}_{i} \cdot \boldsymbol{t}_{i}=\partial \Gamma_{i} / \partial s_{i}$ denotes the (true) vortex sheet strength of the interface $I_{i}$ derived from the circulation $\Gamma_{i} \equiv \phi_{i}-\phi_{i-1}$, where $s_{i}$ and $\boldsymbol{t}_{i}$ are length and unit tangent of the interface $I_{i}$, respectively. Here, the subscript $e$ denotes the differentiation with respect to $e$, and $s_{i, e}=\sqrt{X_{i, e}^{2}+Y_{i, e}^{2}}$. We take the principal value P.V. in the integral (5) when the point $\boldsymbol{x}=(x, y)$ is on the interface $I_{i}$; $(x, y)=\left(X_{i}, Y_{i}\right)$. The second terms on the right-hand side of Eq. (5) denote the contribution from the other interface $I_{j}(i \neq j, i, j=1,2)$. It should be noted that we do not need to take the principal value for the integral of these terms. If we set the regularized parameter $\delta^{49,50}$ in Eq. (5) to $\delta=0$ and adopt a suitable integral method, ${ }^{60}$ the numerical results for the spatial integration have the spectral accuracy. ${ }^{46} \mathrm{We}$ mention that since the second integrals in Eq. (5) without the notation P.V. are not singular integrals, the parameter $\delta$ is unnecessary for these integrals in the meaning of the regularization. However, when an interface is so close to the other one (refer to Figs. 10, 12, and 13), the denominators of the integrands in these integrals can be very small when we set $\delta=0$, which causes some numerical instabilities in the last computed stage. In order to avoid that and retain the resolution at the last computed stage of the computation, we also insert $\delta$ in the second integrals of Eq. (5). The velocity $\boldsymbol{W}_{i}$ in Eq. (5) corresponds to the average velocity $\left(\boldsymbol{u}_{i}+\boldsymbol{u}_{i-1}\right) / 2$ between the two fluid velocities $\boldsymbol{u}_{i}$ and $\boldsymbol{u}_{i-1}$.

The fluid velocity $\boldsymbol{W}=\left(W_{x}, W_{y}\right)$ at an arbitrary point $\boldsymbol{x}=(x, y)$ in bulks is given by replacing $X_{i}$ and $Y_{i}$ with the coordinates $x$ and $y$ in Eq. (5). Then, all integrals in the right-hand side of Eq. (5) are regular integrals. Equation (5) is an extension of the BirkhoffRott equation ${ }^{53-55}$ to two interfaces. Taking into account the relation $\boldsymbol{\gamma}_{i}=\boldsymbol{u}_{i}-\boldsymbol{u}_{i-1}$ and $\boldsymbol{\gamma}_{i}=\gamma_{i} \boldsymbol{t}_{i}$, the fluid velocities of the upper and lower sides of the interfaces are given by

$$
\begin{aligned}
& \boldsymbol{u}_{0}=\boldsymbol{W}_{1}-\frac{\gamma_{1}}{2} \boldsymbol{t}_{1}, \\
& \boldsymbol{u}_{11}=\boldsymbol{W}_{1}+\frac{\gamma_{1}}{2} \boldsymbol{t}_{1}, \\
& \boldsymbol{u}_{12}=\boldsymbol{W}_{2}-\frac{\gamma_{2}}{2} \boldsymbol{t}_{2}, \\
& \boldsymbol{u}_{2}=\boldsymbol{W}_{2}+\frac{\gamma_{2}}{2} \boldsymbol{t}_{2},
\end{aligned}
$$


where $\boldsymbol{u}_{11}$ and $\boldsymbol{u}_{12}$ are the velocities of the lower side of interface $I_{1}$ and the upper side of interface $I_{2}$ in fluid region 1 , respectively. It should be noted that the normal component of the velocities is continuous at each interface because $\boldsymbol{\gamma}_{i}\left(\boldsymbol{\gamma}_{i}=\gamma_{i} \boldsymbol{t}_{i}\right)$ does not possess the normal component.

Now, we determine the Lagrange motion of the interfaces. Unlike the case of a single interface, there are two interfacial velocities in the current system associated with those interfaces; therefore, two Lagrange derivatives with respect to time can exist depending on the interfacial velocities. The normal component of the fluid velocity across each interface should always be continuous. However, there is an arbitrariness how to select the tangential velocity at the interfaces. ${ }^{34,45,61-63}$ Here, we define the interfacial velocity $\boldsymbol{u}_{i}^{+}$at each interface $I_{i}$ labeled by the Lagrange parameter $e$ as

$$
\boldsymbol{u}_{i}^{+}=W_{i}+\frac{\tilde{\alpha}_{i}}{2} \gamma_{i}
$$

where $\tilde{\alpha}_{i}\left(\left|\tilde{\alpha}_{i}\right| \leq A_{i}\right)$ is an artificial parameter depending on the Atwood number $A_{i},{ }^{61,64}$ and we set $\tilde{\alpha}_{i}=0$ when $A_{i}=0$. Equation (7) is an extension of the velocity of a single interface ${ }^{34-36,45}$ to the one of two interfaces.

Then, the interface $I_{i}$ moves with the Lagrange derivative $\mathrm{d} / \mathrm{d} t_{i}$,

$$
\frac{\mathrm{d}}{\mathrm{d} t_{i}}=\frac{\partial}{\partial t}+\boldsymbol{u}_{i}^{+} \cdot \nabla=\frac{\mathrm{d}}{\mathrm{d} t}+\frac{\tilde{\alpha}_{i}}{2} \boldsymbol{\gamma}_{i} \cdot \nabla,
$$

where $\mathrm{d} / \mathrm{d} t$ is defined by

$$
\frac{\mathrm{d}}{\mathrm{d} t}=\frac{\partial}{\partial t}+\boldsymbol{u}^{+} \cdot \nabla
$$

The derivative $\mathrm{d} / \mathrm{d} t$ denotes the Lagrange derivative moving with the velocity $\boldsymbol{u}^{+}$, which coincides with $\boldsymbol{W}_{i}$ at interface $I_{i}$,

$$
\left.\boldsymbol{u}^{+}\right|_{\boldsymbol{x}=\boldsymbol{X}_{i}}=\boldsymbol{W}_{i} .
$$

The parameter $\tilde{\alpha}_{i}$ in Eq. (7) controls the magnitude of the tangential velocity on the interface. ${ }^{45,61,64}$ When the regularized parameter $\delta$ $=0$, we select $\tilde{\alpha}_{i}$ as $\tilde{\alpha}_{i}=-A_{i}$, for which the interfacial velocities $\boldsymbol{u}_{1}^{+}$ and $\boldsymbol{u}_{2}^{+}$become the weighted averages of two fluids derived from the interfacial velocities (6),

$$
\begin{aligned}
& \boldsymbol{u}_{1}^{+}=\frac{\rho_{0} \boldsymbol{u}_{0}+\rho_{1} \boldsymbol{u}_{11}}{\rho_{0}+\rho_{1}}, \\
& \boldsymbol{u}_{2}^{+}=\frac{\rho_{1} \boldsymbol{u}_{12}+\rho_{2} \boldsymbol{u}_{2}}{\rho_{1}+\rho_{2}},
\end{aligned}
$$

while we select $\tilde{\alpha}_{i}$ as $\tilde{\alpha}_{i}=-A_{i}^{2}$ when $\delta \neq 0^{34,58,59}$ throughout this paper, by which we can suppress the rapid increase in the tangential velocity in rolling-up of the vortex sheet at the late stage. ${ }^{61}$

Rewriting the Bernoulli equation (3) using the velocity (7), we obtain the following equation at interface $I_{i}$ :

$$
\begin{aligned}
\frac{\mathrm{d} \Gamma_{i}}{\mathrm{~d} t_{i}}= & 2 A_{i} \frac{\mathrm{d} \Phi_{i}}{\mathrm{~d} t_{i}}+\left(\boldsymbol{u}_{i}^{+}-\boldsymbol{W}_{i}\right) \cdot \nabla \Gamma_{i}-2 A_{i}\left(\boldsymbol{u}_{i}^{+} \cdot \nabla\right) \Phi_{i} \\
& +A_{i}\left(\boldsymbol{W}_{i} \cdot \boldsymbol{W}_{i}+\frac{1}{4} \nabla \Gamma_{i} \cdot \nabla \Gamma_{i}\right)
\end{aligned}
$$

where $\Phi_{i}=\left(\phi_{i-1}+\phi_{i}\right) / 2(i=1,2), \nabla \Phi_{1}=\left(\boldsymbol{u}_{0}+\boldsymbol{u}_{11}\right) / 2$, and $\nabla \Phi_{2}$ $=\left(\boldsymbol{u}_{12}+\boldsymbol{u}_{2}\right) / 2$. Differentiating (10) with respect to $e$ and using the
Lagrange derivatives (8) and (9), we obtain the evolution equation for the sheet strength $\gamma_{i}=\boldsymbol{t}_{i} \cdot \nabla \Gamma_{i}$ described by a single derivative $\mathrm{d} / \mathrm{d} t$,

$$
\begin{aligned}
\frac{\mathrm{d} \gamma_{i}}{\mathrm{~d} t}= & \frac{2 A_{i}}{s_{i, e}}\left(X_{i, e} \frac{\mathrm{d} W_{i, x}}{\mathrm{~d} t}+Y_{i, e} \frac{\mathrm{d} W_{i, y}}{\mathrm{~d} t}\right) \\
& -\frac{\left(1+\tilde{\alpha}_{i} A_{i}\right) \gamma_{i}}{s_{i, e}^{2}}\left(X_{i, e} W_{i, x, e}+Y_{i, e} W_{i, y, e}\right)+\frac{A_{i}-5 \tilde{\alpha}_{i}}{4 s_{i, e}}\left(\gamma_{i}^{2}\right)_{e} \\
& +\frac{A_{i} \tilde{\alpha}_{i}}{s_{i, e}}\left(\gamma_{i, e} T_{i}+\gamma_{i} T_{i, e}\right)
\end{aligned}
$$

where $T_{i}=\boldsymbol{t}_{i} \cdot \boldsymbol{W}_{i}$, and we set the temporal evolution of interface $I_{i}$ using Eq. (7) as

$$
\frac{\mathrm{d} \boldsymbol{X}_{i}}{\mathrm{~d} t}=\boldsymbol{u}_{i}^{+}
$$

Solving Eqs. (11) and (12) simultaneously by taking Eqs. (5) and (7) into account, we can determine the motion of interfaces $I_{1}$ and $I_{2}$ together with the bulk velocity field.

\section{LINEAR ANALYSIS AND INITIAL CONDITIONS FOR NUMERICAL CALCULATIONS}

\section{A. Linear analysis for the double-layer solution}

In this subsection, we perform the linear analysis in order to determine the initial conditions for numerical calculations. We denote the interface as $y=\eta_{i}(x, t)$. The kinematic boundary conditions at interface $I_{i}(i=1,2)$ are given by

$$
\begin{aligned}
& \frac{\partial \eta_{1}}{\partial t}-\frac{\partial \phi_{i}}{\partial y}=\frac{\partial \phi_{i}}{\partial x} \frac{\partial \eta_{1}}{\partial x} \quad(i=0,1) \quad \text { at } I_{1}, \\
& \frac{\partial \eta_{2}}{\partial t}-\frac{\partial \phi_{i}}{\partial y}=\frac{\partial \phi_{i}}{\partial x} \frac{\partial \eta_{2}}{\partial x} \quad(i=1,2) \quad \text { at } I_{2} .
\end{aligned}
$$

We assume that the non-perturbative states of interfaces $I_{1}$ and $I_{2}$ are $y=d / 2$ and $y=-d / 2(d>0)$, respectively (refer to Fig. 1), and consider small deviations from those states. Linearizing the Bernoulli equation (3) and the kinematic boundary condition (13) at the non-perturbative interfaces, we obtain the following linearized equations at $I_{1}(y=d / 2)$ :

$$
\begin{gathered}
\rho_{0} \frac{\partial \tilde{\phi}_{0}}{\partial t}=\rho_{1} \frac{\partial \tilde{\phi}_{1}}{\partial t}, \\
\frac{\partial \tilde{\eta}_{1}}{\partial t}=\frac{\partial \tilde{\phi}_{0}}{\partial y}, \quad \frac{\partial \tilde{\eta}_{1}}{\partial t}=\frac{\partial \tilde{\phi}_{1}}{\partial y},
\end{gathered}
$$

and at $I_{2}(y=-d / 2)$,

$$
\begin{gathered}
\rho_{1} \frac{\partial \tilde{\phi}_{1}}{\partial t}=\rho_{2} \frac{\partial \tilde{\phi}_{2}}{\partial t}, \\
\frac{\partial \tilde{\eta}_{2}}{\partial t}=\frac{\partial \tilde{\phi}_{1}}{\partial y}, \quad \frac{\partial \tilde{\eta}_{2}}{\partial t}=\frac{\partial \tilde{\phi}_{2}}{\partial y}
\end{gathered}
$$

where the quantities with tilde denote small perturbations: $\left|\tilde{\eta}_{i}\right| \ll 1$ $(i=1,2)$ and $\left|\tilde{\phi}_{i}\right| \ll 1(i=0,1,2)$. 
Taking into account the Laplace field (2) and the periodicity in the $x$ direction, we have the following linear solution to Eqs. (14) and (15):

$$
\begin{aligned}
& \tilde{\eta}_{1}=a_{1} t \cos k x+\frac{d}{2}, \\
& \tilde{\eta}_{2}=a_{2} t \cos k x-\frac{d}{2}, \\
& \tilde{\phi}_{0}=B_{0} \mathrm{e}^{-k y} \cos k x \quad\left(y>\frac{d}{2}\right), \\
& \tilde{\phi}_{1}=\left(B_{11} e^{k y}+B_{12} \mathrm{e}^{-k y}\right) \cos k x \quad\left(-\frac{d}{2} \leq y \leq \frac{d}{2}\right), \\
& \tilde{\phi}_{2}=B_{2} \mathrm{e}^{k y} \cos k x \quad\left(y<-\frac{d}{2}\right),
\end{aligned}
$$

where $k$ is the wavenumber, and we can select the amplitudes $a_{1}$ and $a_{2}$ independently. Using $a_{i}(i=1,2)$, the coefficients $B_{0}, B_{11}, B_{12}$, and $B_{2}$ are given as

$$
\begin{aligned}
B_{0} & =-\frac{a_{1}}{k} \mathrm{e}^{k d / 2}, \\
B_{11} & =\frac{a_{1} \mathrm{e}^{k d / 2}-a_{2} \mathrm{e}^{-k d / 2}}{k\left(\mathrm{e}^{k d}-\mathrm{e}^{-k d}\right)}, \\
B_{12} & =\frac{a_{1} \mathrm{e}^{-k d / 2}-a_{2} \mathrm{e}^{k d / 2}}{k\left(\mathrm{e}^{k d}-\mathrm{e}^{-k d}\right)}, \\
B_{2} & =\frac{a_{2}}{k} \mathrm{e}^{k d / 2} .
\end{aligned}
$$

Following the case of the conventional single-layer RMI, we normalize the physical quantities in Eq. (16) by the wavenumber $k$ and the initial velocity shear of a single interface $v_{l i n}$, which corresponds to the linear growth rate in the single-layer RMI. ${ }^{21,24,25,58,59}$ From now on, the dimensionless variables for space $k x$, time $k v_{\text {lin }} t$, circulation of vortex sheet $i k \Gamma_{i} / v_{\text {lin }}(i=1,2)$, and vortex sheet strength $\gamma_{i} / v_{\text {lin }}$ are used as $\boldsymbol{x}, t, \Gamma_{i}$, and $\gamma_{i}$. The dimensionless distance $k d$ between the interfaces is replaced with $d$ below. Then, from Eqs. (16) and (17), we have the normalized linear solution

$$
\begin{gathered}
\tilde{\eta}_{1}=a_{1} t \cos x+\frac{d}{2}, \\
\tilde{\eta}_{2}=a_{2} t \cos x-\frac{d}{2}, \\
\tilde{\phi}_{0}=-a_{1} \mathrm{e}^{d / 2-y} \cos x \quad\left(y>\frac{d}{2}\right), \\
\tilde{\phi}_{1}=\left[\frac{a_{1} \mathrm{e}^{d / 2+y}-a_{2} \mathrm{e}^{-d / 2+y}}{\mathrm{e}^{d}-\mathrm{e}^{-d}}+\frac{a_{1} \mathrm{e}^{-d / 2-y}-a_{2} \mathrm{e}^{d / 2-y}}{\mathrm{e}^{d}-\mathrm{e}^{-d}}\right] \cos x \\
\times\left(-\frac{d}{2} \leq y \leq \frac{d}{2}\right), \\
\tilde{\phi}_{2}=a_{2} \mathrm{e}^{d / 2+y} \cos x \quad\left(y<-\frac{d}{2}\right) .
\end{gathered}
$$

The linear solution [Eq. (18)] provides the initial conditions for solving the nonlinear interaction between the two interfaces.

\section{B. Initial conditions for numerical calculations}

Throughout this paper, we set the initial interfacial shapes as

$$
\begin{array}{ll}
I_{1}: & X_{1}(e, 0)=e, \quad Y_{1}(e, 0)=\frac{d}{2}, \\
I_{2}: & X_{2}(e, 0)=e, \quad Y_{2}(e, 0)=-\frac{d}{2}
\end{array}
$$

for all numerical calculations $(-\pi \leq e \leq \pi)$.

For the linear solution (18) and the initial condition (19), we obtain the initial vortex sheet strengths $\gamma_{1}=\left.\left(\partial \phi_{1} / \partial x-\partial \phi_{0} / \partial x\right)\right|_{y=d / 2}$ $\left[s_{1, e}(e, 0)=1\right]$ and $\gamma_{2}=\left.\left(\partial \phi_{2} / \partial x-\partial \phi_{1} / \partial x\right)\right|_{y=-d / 2}\left[s_{2, e}(e, 0)=1\right]$ as

$$
\begin{aligned}
& \gamma_{1}(e, 0)=-\frac{2\left(a_{1} \mathrm{e}^{d}-a_{2}\right)}{\mathrm{e}^{d}-\mathrm{e}^{-d}} \sin e, \\
& \gamma_{2}(e, 0)=-\frac{2\left(a_{2} \mathrm{e}^{d}-a_{1}\right)}{\mathrm{e}^{d}-\mathrm{e}^{-d}} \sin e .
\end{aligned}
$$

When we approach interface $I_{2}$ toward interface $I_{1}(d \rightarrow 0)$, we have

$$
\begin{aligned}
& \lim _{d \rightarrow 0} \gamma_{1}=\left[\frac{-2 a_{1} \mathrm{e}^{d}}{e^{d}+\mathrm{e}^{d}}\right]_{d=0} \sin e=-a_{1} \sin e, \\
& \lim _{d \rightarrow 0} \gamma_{2}=\left[\frac{-2 a_{2} \mathrm{e}^{d}}{e^{d}+\mathrm{e}^{d}}\right]_{d=0} \sin e=-a_{2} \sin e,
\end{aligned}
$$

where we used L'Hôpital's rule. Equation (21) gives the limit sheet strength

$$
\gamma=\lim _{d \rightarrow 0}\left(\gamma_{1}+\gamma_{2}\right)=\left[\frac{\partial \phi_{2}}{\partial x}-\frac{\partial \phi_{0}}{\partial x}\right]_{d=0}=-\left(a_{1}+a_{2}\right) \sin e .
$$

This $\gamma$ coincides with the one in the conventional single-layer RMI when $a_{1}=a_{2}=1$. $^{17,3}$

From now on, we normalize the Atwood numbers $A_{i}(i=1,2)$ in (4) with the fluid density $\rho_{1}\left(\rho_{1} \neq 0\right)$ as follows:

$$
A_{1}=\frac{\tilde{\rho}_{0}-1}{1+\tilde{\rho}_{0}}, \quad A_{2}=\frac{1-\tilde{\rho}_{2}}{1+\tilde{\rho}_{2}}
$$

where $\tilde{\rho}_{0}=\rho_{0} / \rho_{1}$ and $\tilde{\rho}_{2}=\rho_{2} / \rho_{1}$. Depending on the density ratios, we consider the following two cases:

$$
\begin{gathered}
\rho_{0}<\rho_{1}<\rho_{2} \text {, i.e., } \tilde{\rho}_{0}<1 \text { and } \tilde{\rho}_{2}>1, \\
\rho_{0}<\rho_{1} \text { and } \rho_{2}<\rho_{1} \text {, i.e., } \tilde{\rho}_{0}<1 \text { and } \tilde{\rho}_{2}<1 .
\end{gathered}
$$

Case (23b) corresponds to the situation investigated by Liu et al. ${ }^{8}$ using the weakly nonlinear analysis when $\rho_{1}=\rho_{2}=0$ (the vacuums). The situation that was investigated numerically by Zabusky and Zhang ${ }^{41}$ is also case (23b), in which they set $\rho_{0}=\rho_{2}$ and $\rho_{0} / \rho_{1}$ $=0.14$. The cases that $\rho_{0}>\rho_{1}$ and $\rho_{2}>\rho_{1}$, i.e., $\tilde{\rho}_{0}>1$ and $\tilde{\rho}_{2}>1$, are also possible, but this is essentially the same as case (23b) for RMI in the planar geometry because we do not consider the gravity in the current study.

Here, we normalize the linear amplitude $a_{i}(i=1,2)$ as $\left|a_{i}\right|=1$. Then, there are two options for $a_{1}$ and $a_{2}$ : one of which is in phase,

$$
\frac{a_{2}}{a_{1}}=1
$$


and the other is out of phase,

$$
\frac{a_{2}}{a_{1}}=-1
$$

where Eq. (24) provides the initial limit sheet strength of the conventional single-layer RMI: $\gamma=\lim _{d \rightarrow 0}\left(\gamma_{1}+\gamma_{2}\right)_{t=0}=-2 \sin e^{21,34-36,58,59}$ when $a_{1}=a_{2}=1$, while $\gamma=\lim _{d \rightarrow 0}\left(\gamma_{1}+\gamma_{2}\right)_{t=0}=0$ for Eq. (25).

We compare the numerical results for double-layer interfaces with those for the conventional single-layer interface, in which the Atwood number in the latter case is defined as

$$
A=\frac{\rho_{2}-\rho_{1}}{\rho_{1}+\rho_{2}},
$$

where $\rho_{2}>\rho_{1}$ is assumed for two fluid components 1 and 2 (fluid 1 is upper). The initial condition for the single-layer interface $(X, Y)$ and the initial sheet strength $\gamma$ are selected as

$$
\begin{aligned}
& X(e, 0)=e \quad(-\pi \leq e \leq \pi), \\
& Y(e, 0)=0, \\
& \gamma(e, 0)=-2 \sin e
\end{aligned}
$$

for both cases of the regularized parameter $\delta=0$ and $\delta \neq 0$.

\section{NUMERICAL CALCULATIONS BY VSM}

In this section, we perform numerical calculations based on the governing equations (11) and (12) with Eqs. (5) and (7) and investigate the nonlinear interaction between the two interfaces in the double-layer RMI.

\section{A. Overview of numerical methods}

In this subsection, we briefly mention the numerical methods adopted in the current study. When the regularized parameter $\delta=0$, we adopt the alternate point quadrature method ${ }^{34,46,60}$ for the spatial integration of Eq. (5). This scheme is known to be spectral accuracy. ${ }^{46}$ When $\delta \neq 0$, we adopt the conventional trapezoidal rule for the spatial integration. ${ }^{57}$ For the temporal integration, we use the fourth-order Runge-Kutta scheme throughout this paper for both calculations of $\delta=0$ and $\delta \neq 0$. The simultaneous Fredholm equations of the second kind [Eq. (11)] are solved by iteration with a tolerance level of $10^{-12}$.

In order to prevent the irregular motion due to the round-off error, we use the filtering technique (filter levels $10^{-11}$ for $\delta=0$ and $10^{-13}$ for $\delta \neq 0$ ) introduced by Krasny. ${ }^{49}$ The growth of higher-order Fourier modes in calculations of multi-layer interfaces is more rapid than that of single-layer RMI, especially for the calculations of $\delta=0$, and the above Krasny's filter is not enough to suppress the growth when $\delta=0$. To control the higher-order Fourier modes, we adopt the following 16th-order Fourier exponential filter: :3,65,66 $^{-6}$

$$
\exp \left[-10\left(\frac{|k|}{N / 2}\right)^{16}\right] \hat{f}(k)
$$

for mode $k$ and the number of grid points $N$ to damp the highest modes, where $\hat{f}(k)$ is the Fourier transform of an arbitrary function $f(e)$. The exponential filter (27) is only employed for the calculations of $\delta=0$.
When $\delta \neq 0$, we adopt the grid redistribution method by use of Newton's method to avoid clustering ${ }^{61}$ of grid points. ${ }^{17,34,67}$ There is another numerical method to avoid the clustering, which is known as the point insertion scheme. ${ }^{49,58,59,68}$ This method can capture the complicated structure of the vortex sheet at the late stage by inserting grid points successively; however, the roll-up of the vortex sheet is too strong, and the asymptotic growth rate of the bubble and spike deviates ${ }^{68}$ from the value obtained by the theoretical prediction. ${ }^{40,69}$ In addition to that, the point insertion scheme is unsuitable for the calculation of high Atwood numbers. For more details on the numerical schemes adopted here, refer to Refs. 17 and 34.

\section{B. Numerical results for $\delta=0$}

In this subsection, we present the numerical results by the VSM for $\delta=0$ in Eq. (5) and compare those with the ones of the singlelayer RMI. In numerical calculations of $\delta=0$, the number of grid points $N$ taken on the interfaces is selected as $N=1024$. We set the normalized time step $\Delta t$ as $\Delta t=10^{-4}$ throughout the calculations for $\delta=0$. In this subsection, we investigate the situation of Eq. (23b) for two types of density ratios, large $\left(\left|A_{i}\right|=1\right)$ and small $\left(\left|A_{i}\right|=0.2\right)$, one of which is $\tilde{\rho}_{0}=0$ and $\tilde{\rho}_{2}=0$, i.e., $A_{1}=-1$ and $A_{2}=1$ in Eq. (22), which corresponds to the situation that fluid 0 and fluid 2 are the vacuums, and the other is $\tilde{\rho}_{0}=\tilde{\rho}_{2}=2 / 3$, i.e., $A_{1}=-0.2$ and $A_{2}$ $=0.2$ in Eq. (22). We also compare the numerical results with those for $A=1$ and $A=0.2$ in the single-layer RMI. Throughout this subsection, we consider the in-phase situation [Eq. (24)] $\left(a_{1}=a_{2}=1\right)$. We select the initial distance $d$ between two interfaces in Eq. (19) as $d=\pi\left(d=\lambda / 2\right.$, where $\lambda$ is the wavelength) when $A_{1}=-1$ and $A_{2}=1$. For the case of $A_{1}=-0.2$ and $A_{2}=0.2$, we consider two cases of $d$ $=\pi$ and $d=\pi / 4$.

Figure 2 shows the interfacial structures and the velocity fields for (left) $A_{1}=-1$ and $A_{2}=1$ in Eq. (22) $\left(\rho_{0}=\rho_{2}=0\right)$ and (right) $A$ $=1$ (the upper fluid $\rho_{1}=0$ ). The points $x=0$ on the upper interface $I_{1}$ and $x= \pm \pi$ on the lower interface $I_{2}$ correspond to spikes, and the points $x= \pm \pi$ on $I_{1}$ and $x=0$ on $I_{2}$ correspond to bubbles in the left panel, while the point $x=0(x= \pm \pi)$ corresponds to the spike (bubble) in the right panel. The interfacial shape in the neighborhood of
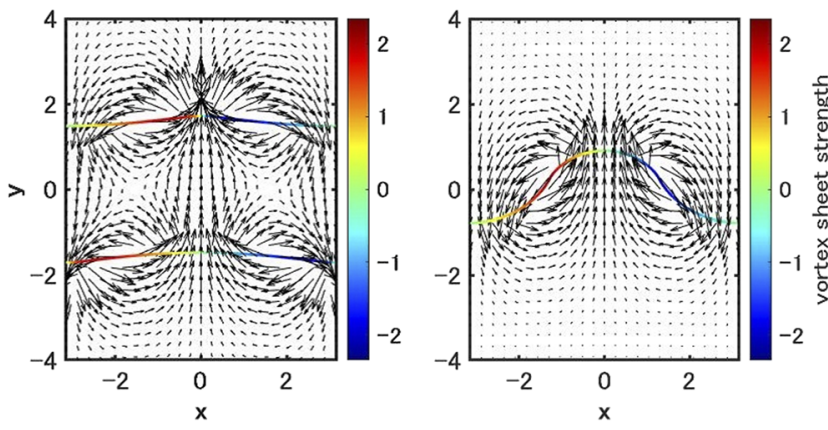

FIG. 2. Interfacial structures for $\delta=0$ with the colored scale of the vortex sheet strengths and the velocity fields for the (left) double-layer RMI and (right) singlelayer RMI, where the Atwood numbers are (left) $A_{1}=-1$ and $A_{2}=1$ and (right) $A=1$. Both panels show the last computed stage of the evolution for those Atwood numbers, where $t=0.12$ for the left panel and $t=0.55$ for the right panel. The initial distance $d$ between $I_{1}$ and $I_{2}$ in the left panel is selected as $d=\pi$. 
the bubble ( $x= \pm \pi$ on $I_{1}$ and $x=0$ on $I_{2}$ ) and spike ( $x=0$ on $I_{1}$ and $x= \pm \pi$ on $I_{2}$ ) is the same in the two interfaces. We see that two stagnation points exist in the velocity field at around $x= \pm \pi / 2$ and $y$ $=0$ in the left panel. The change in the distance between two interfaces is not so remarkable for the case of $A_{1}=-1$ and $A_{2}=1$.

In the numerical calculations, the interfacial coordinate $\left(X_{i}, Y_{i}\right)$ $(i=1,2)$ is expanded using Fourier modes ${ }^{34,46}$

$$
\begin{aligned}
& X_{i}(e, t)=e+\sum_{k=-N / 2+1}^{N / 2} \hat{X}_{i, k}(t) \mathrm{e}^{i k e}, \\
& Y_{i}(e, t)=\sum_{k=-N / 2+1}^{N / 2} \hat{Y}_{i, k}(t) \mathrm{e}^{i k e} .
\end{aligned}
$$

We show the growth of the Fourier amplitude $\sqrt{\hat{X}_{1}(t)^{2}+\hat{Y}_{1}(t)^{2}}$ for $A_{1}=-1$ and $A_{2}=1$ and the corresponding Fourier amplitude of the single-layer RMI for $A=1$ in Fig. 3. We depict the Fourier amplitude for $I_{1}$ in Fig. 3 , but the amplitude for $I_{2}$ at the corresponding
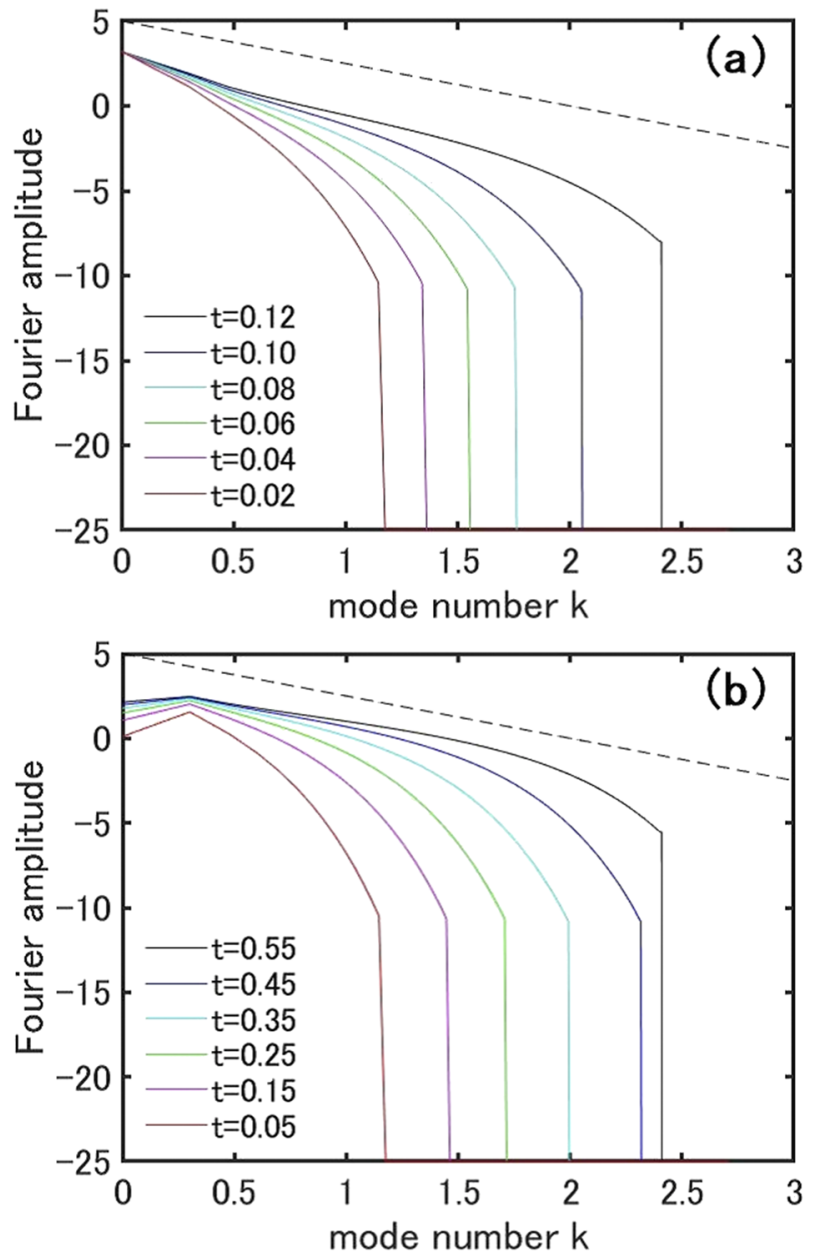

FIG. 3. Fourier amplitudes for $l_{1}$ in the (a) double-layer interfaces and (b) singlelayer interface, where the Atwood numbers are the same as in Fig. 2. The slope of the dotted line is $-5 / 2$. time coincides with the one for $I_{1}$. The dotted lines in (a) and (b) in Fig. 3 possess slope $-5 / 2$, the value obtained by Moore ${ }^{70}$ for KHI. It is known that the curvature singularity occurs as the spectra approach the $-5 / 2$ lines. ${ }^{34,46,70,71}$ This is confirmed in Fig. 4.

Curvatures of $I_{i}(i=1,2)$ at $t=0.12$ and the one of the singlelayer interface at $t=0.55$ are depicted as a function of the Lagrange parameter $e$ in Fig. 4. Although the interfacial structures in Fig. 2 are sufficiently smooth, the corresponding curvatures in Fig. 4 have cusps at the spikes [ $e=0$ on $I_{1}$ and $e= \pm \pi$ on $I_{2}$ in Fig. 4(a) and $e$ $=0$ in Fig. 4(b)]. We mention that the vortex sheet strength $\gamma_{i}(\gamma)$ in the neighborhood of the spike is not so large; however, the derivative $\gamma_{i, e}\left(\gamma_{e}\right)$ is quite large because the sheet strength $\gamma_{i}(\gamma)$ changes its sign at the spike (refer to Fig. 2). The numerical calculations for these Atwood numbers break down immediately after $\left(t=t_{c}=+0.12\right.$ for the double-layer RMI and $t=t_{c}=0.55$ for the single-layer RMI) the appearance of the curvature singularity. From now on, we refer to $t_{c}$ as the break-down time.
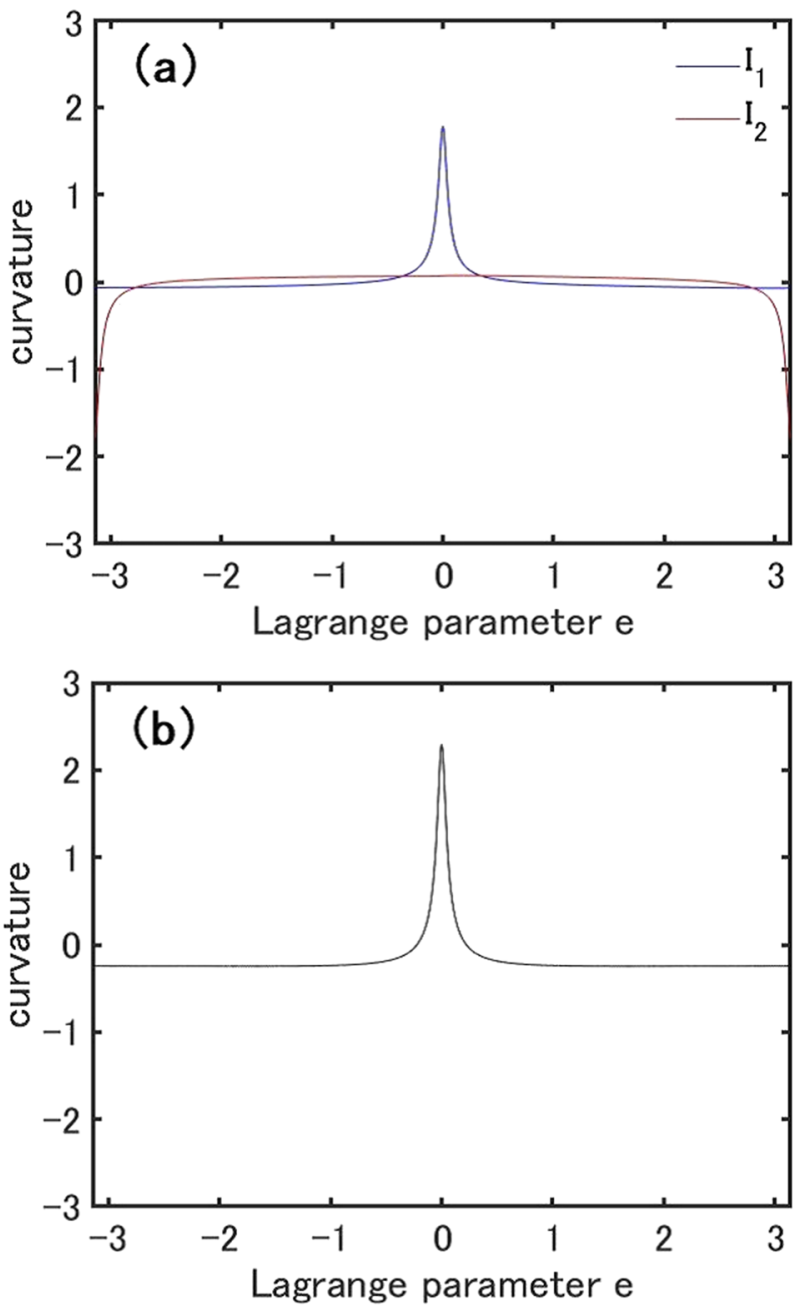

FIG. 4. Curvatures of (a) $I_{1}$ (blue line) and $I_{2}$ (red line) in the double-layer interfaces at $t=0.12$ and $(\mathrm{b})$ single-layer interface at $t=0.55$, where the Atwood numbers are the same as in Figs. 2 and 4. 

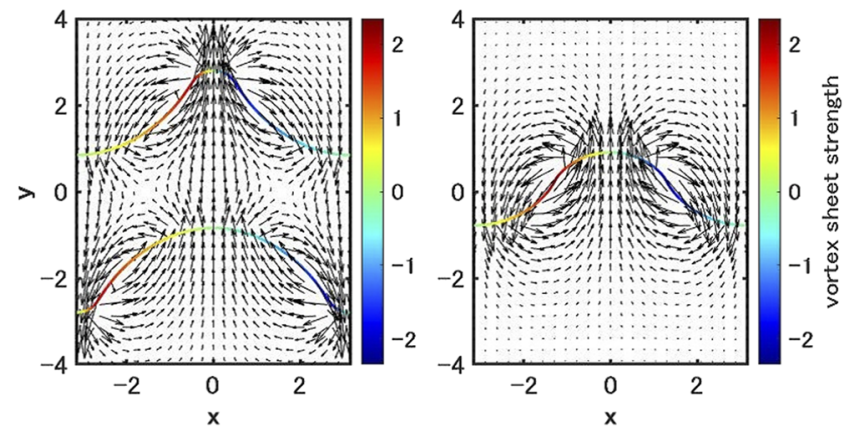

FIG. 5. Interfacial structures for $\delta=0$ with the colored scale of the vortex sheet strengths and the velocity fields for the (left) double-layer RMI and (right) singlelayer RMI, where the Atwood numbers are (left) $A_{1}=-0.2$ and $A_{2}=0.2$ and (right) $A=0.2$. Both panels show the last computed stage of the evolution for those Atwood numbers, where $t=1.0$ for the left panel and $t=0.94$ for the right panel. The initial distance $d$ between $I_{1}$ and $I_{2}$ in the left panel is selected as $d=\pi$.

Figure 5 shows the interfacial structures and the velocity fields for (left) $A_{1}=-0.2$ and $A_{2}=0.2$ in Eq. (22) $\left(\rho_{0}=\rho_{2}=2 / 3\right)$ and (right) $A=0.2$ (the upper fluid is lighter). The locations of bubbles and spikes are the same as those in Fig. 2. Both of the deformations of bubbles and spikes for $A_{1}=-0.2$ and $A_{2}=0.2$ are large compared to those for $A_{1}=-1$ and $A_{2}=1$ (Fig. 2), although the vortex sheet strengths $\gamma_{1}$ and $\gamma_{2}$ in the double-layer RMI in Fig. 5 are weaker than the ones in Fig. 2 or those in the right panel of Fig. 5. We see that a strong upward (downward) velocity field exists around the spike at $x=0$ on $I_{1}\left(x= \pm \pi\right.$ on $\left.I_{2}\right)$, and the shape of spikes of the double-layer RMI (left panel) becomes sharper than the one of the single-layer RMI (right panel) for these small Atwood numbers $\left(\left|A_{i}\right|=A=0.2\right)$.

We depict the Fourier amplitude for $I_{1}$ (the one for $I_{2}$ at the corresponding time is the same) for $A_{1}=-0.2$ and $A_{2}=0.2$ and the Fourier amplitude of the single-layer RMI for $A=0.2$ in Fig. 6. As the Fourier amplitudes approach the $-5 / 2$ lines, the cusps appear in the curvatures for both the double-layer RMI and the single-Layer RMI, as shown in Fig. 7. These cusps appeared at the spikes for both of the double-layer RMI and the single-layer RMI in Fig. 4. On the other hand, they appear at different places in (a) and (b), in which two cusps appear on both sides of spikes $\left(e=0\right.$ on $I_{1}$ and $e= \pm \pi$ on $\left.I_{2}\right)$ in Fig. $7(\mathrm{a})$, while those appear in the neighborhood of $e= \pm 1$ in Fig. 7(b). At both of which, the vortex sheet strengths $\left|\gamma_{i}\right|(i=1,2)$ and $|\gamma|$ take their maximum values. Unlike the case of $\left|A_{i}\right|=A=1(i=1,2)$, the break-down time of numerical calculations due to the appearance of curvature singularity for the double-layer $\operatorname{RMI}\left(t_{c}=1.0\right)$ is larger than that for the single-layer RMI $\left(t_{c}=0.94\right)$. This break-down time is further extended for $\delta=0$ when the initial distance between the two interfaces $d$ becomes small. We see that in Figs. 7-9.

Figure 8 shows the interfacial structures and the velocity fields of the double-layer RMI with the same Atwood numbers as Fig. 5 and a different initial distance $d=\pi / 4$, where the left panel shows the state of $t=1.0$ and the right panel shows $t=2.0$. As we see from Fig. 8, the vortex sheet strength $\left|\gamma_{i}\right|(i=1,2)$ with this narrow initial distance does not grow, and the concentration of $\left|\gamma_{i}\right|$ as observed in Figs. 2 and 5 is not found in Fig. 8. We see that the distance between
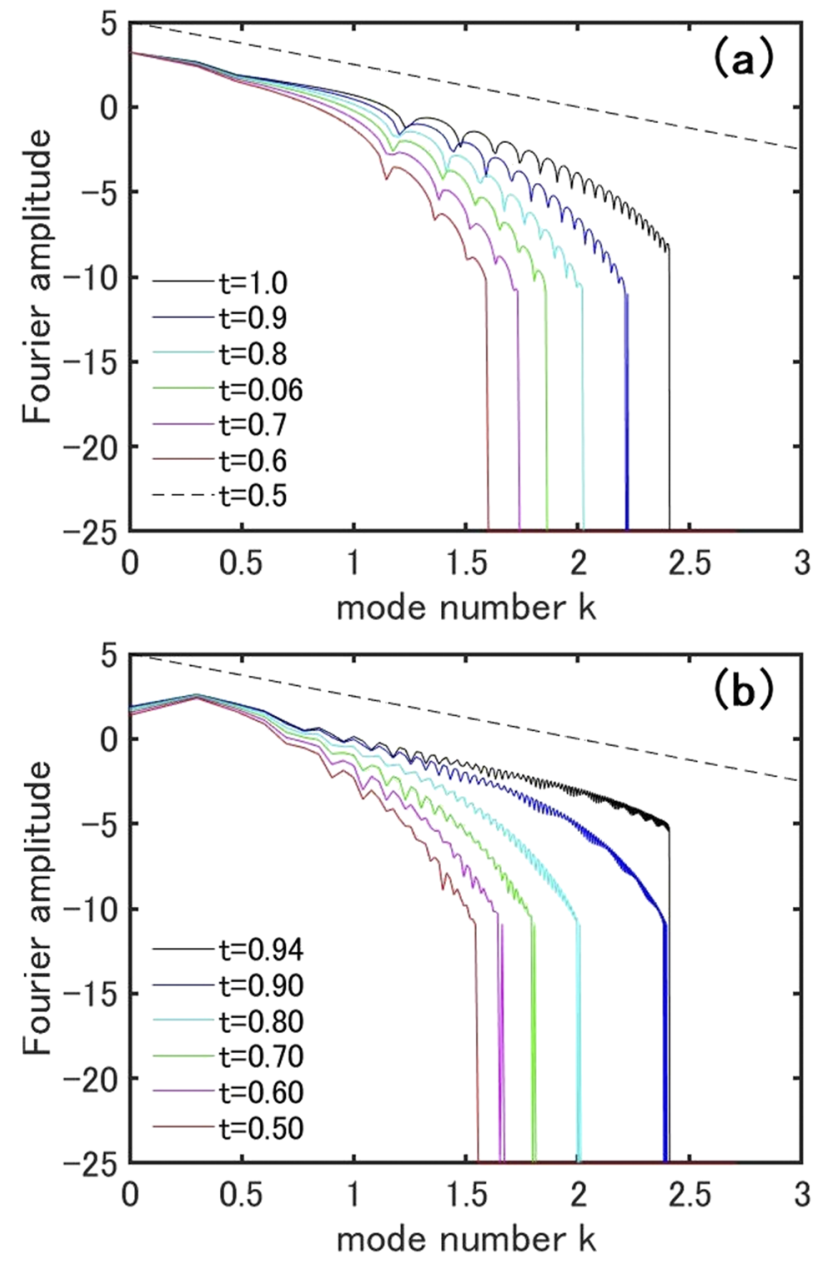

FIG. 6. Fourier amplitudes for $l_{1}$ in the (a) double-layer interfaces and (b) singlelayer interface, where the Atwood numbers are the same as in Fig. 5. The slope of the dotted line is $-5 / 2$.

the two interfaces gradually becomes narrow with the passage of time. The fact that the concentration of the sheet strength $\left|\gamma_{i}\right|$ does not occur is also reflected in the growth of Fourier amplitudes and curvatures of the interfaces.

The upper panel of Fig. 9 shows the Fourier amplitude for $I_{1}$ (the one for $I_{2}$ at the corresponding time is the same) with $A_{1}=-0.2$ and $A_{2}=0.2$ and the initial distance $d=\pi / 4$. The Fourier amplitude with $d=\pi / 4$ does not grow, and it does not attain the line of slope $-5 / 2$ (the dotted line), as shown in Figs. 3 and 6. The curvatures of $I_{1}$ (blue line) and $I_{2}$ (red line) at $t=2.0$ are depicted in the lower panel of Fig. 9 as a function of the Lagrange parameter $e$. Both curvatures of $I_{1}$ and $I_{2}$ are smooth, and the cusps do not appear even at this time. When $t>2.0$, the higher-order Fourier modes grow, and the calculation becomes unstable; however, the noticeable curvature singularity as shown in Figs. 4 and 7 was not observed even at later times. We mention that the calculations for $d=\pi / 4$ are stable for a long time compared to those for $d=\pi$ regardless of the Atwood numbers when $\delta=0$. 

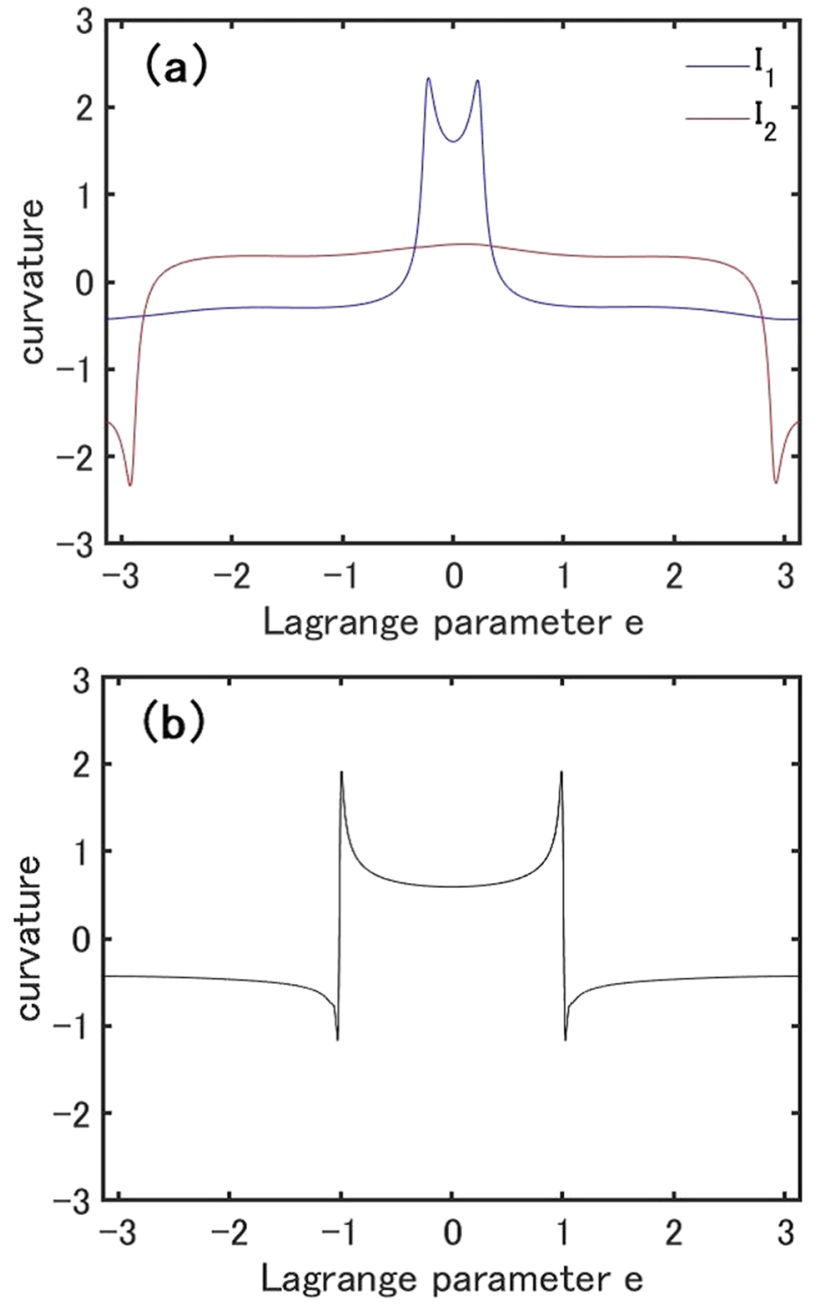

FIG. 7. Curvatures of (a) $I_{1}$ (blue line) and $I_{2}$ (red line) in the double-layer interfaces at $t=1.0$ and (b) single-layer interface at $t=0.94$, where the Atwood numbers are the same as in Figs. 5 and 6.
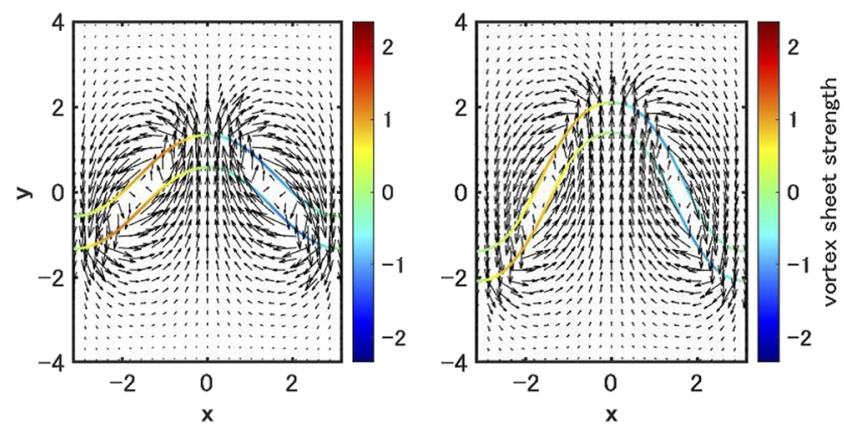

FIG. 8. Interfacial structures for $\delta=0$ with the colored scale of the vortex sheet strengths and the velocity fields of the double-layer RMI for the initial distance $d=\pi / 4$ between the two interfaces, where the Atwood numbers are the same as in Fig. 5. The left and right panels show the velocity fields at $t=1.0$ and $t=2.0$, respectively.
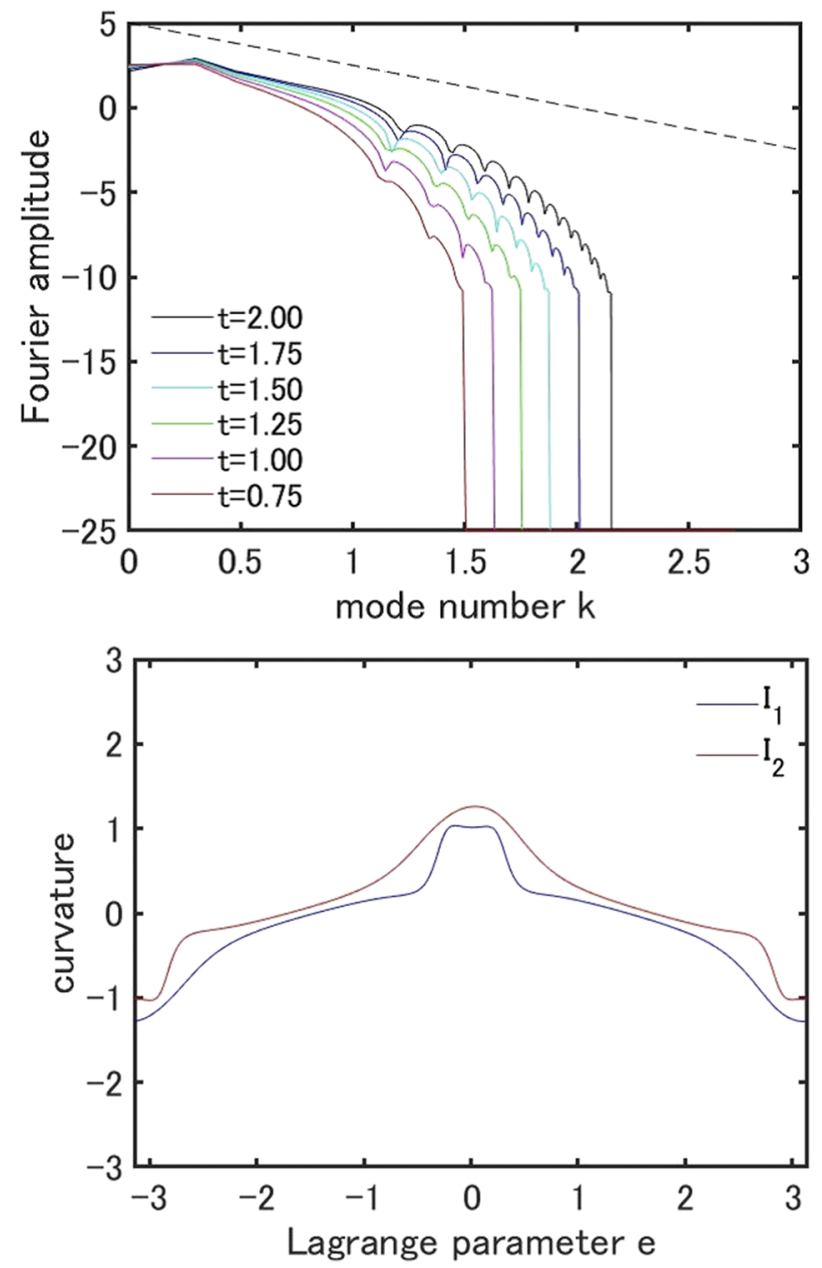

FIG. 9. Fourier amplitude (upper panel) and the curvature at $t=2.0$ (lower panel) in the double-layer interfaces with the initial distance $d=\pi / 4$. The Atwood numbers are the same as in Figs. 5-8. The slope of the dotted line in the upper panel is $-5 / 2$.

\section{Numerical results for finite $\delta$}

In this subsection, we present the numerical results by the VSM for $\delta \neq 0$ using the vortex (blob) method. ${ }^{49,50}$ The calculation for $\delta \neq 0$ does not possess the spectral accuracy as for the calculation in $\delta=0$ presented in Subsection IV B; however, it enables us to compare the numerical results to the long-time behavior in numerical simulations as presented by Zabusky and Zhang. ${ }^{41}$ We adopt the regularized parameter $\delta$ as $\delta=0.15$ throughout this subsection (for the details of the selection of this value, refer to Ref. 58). In numerical calculations, the number of grid points on the interfaces $N$ is selected as $N=512$, and we set the normalized time step $\Delta t$ as $\Delta t=2.5 \times 10^{-3}$ for the calculations of $\delta=0.15$. In this subsection, we consider the nonlinear interactions between the two interfaces for various initial distances $(d=\pi, \pi / 2$, and $\pi / 4)$. We investigate the long-time behavior of the interfaces for the two cases of Eqs. (23a) and (23b), where the absolute value of the Atwood number is fixed as $\left|A_{i}\right|=0.2(i=1,2)$. 
We show the temporal evolution of interfacial structures with the initial distance $d=\pi / 2$ and the linear amplitudes $a_{1}=a_{2}=1$ [in phase (24)] in Fig. 10, where the Atwood numbers are $A_{1}=A_{2}$ $=-0.2\left[\tilde{\rho}_{0}=2 / 3\right.$ and $\tilde{\rho}_{2}=3 / 2$ in Eq. (22)]; that is, we consider the case of $(23 \mathrm{a})$. This is the natural extension of the conventional singlelayer RMI such that the sum of two initial sheet strengths in Eq. (20) becomes equal to the initial sheet strength of the single-layer RMI $\gamma$ in the limit of $d \rightarrow 0: \lim _{d \rightarrow 0}\left(\gamma_{1}(e, 0)+\gamma_{2}(e, 0)\right)=-2 \sin e=\gamma(e$, $0)$. The two interfaces gradually approach each other and merge, and finally, they behave like one interface. This merging occurs for all initial distances $d=\pi$ (refer to Fig. 12), $\pi / 2$, and $\pi / 4$ when $\delta$ is finite. In particular, when the initial distance between two interfaces is small $(d=\pi / 2$ and $d=\pi / 4)$, the two interfaces finally merge including the bubbles $(x= \pm \pi)$ and spikes $(x=0)$, as shown in Fig. 10. The sheet strengths $\gamma_{1}$ and $\gamma_{2}$ concentrate at the vortex cores, ${ }^{72}$ the center of the mushrooms, and the same-signed neighboring cores rotate around each other. The merging phenomenon is caused by the incompressibility of the system that the area-preserving condition must hold. In order to maintain the area of the initial rectangle region between two interfaces, the distance between the interfaces is needed to become
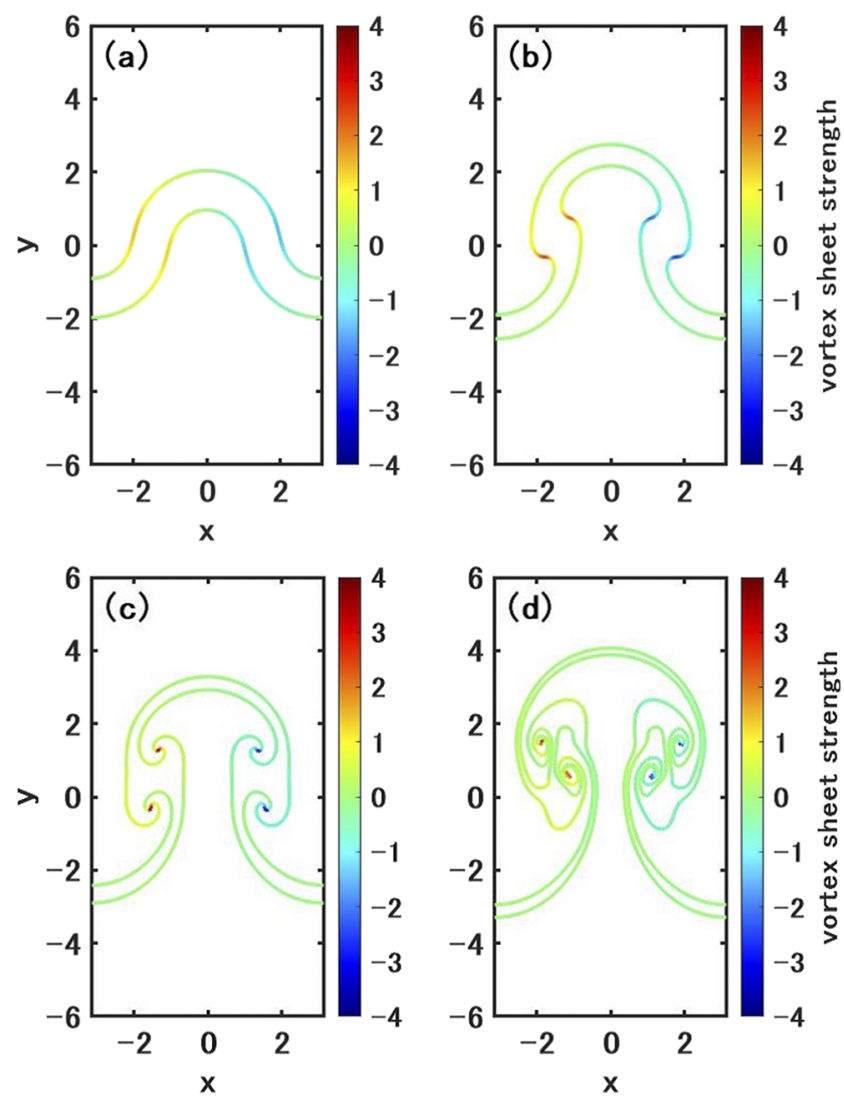

FIG. 10. Temporal evolution of interfacial structures of the double-layer RMI for $\delta$ $=0.15$ with the colored scale of the vortex sheet strengths, where the initial distance $d=\pi / 2$, the Atwood numbers $A_{1}=A_{2}=-0.2$, and the linear amplitudes $a_{1}$ and $a_{2}$ satisfy the in-phase condition (24). The depicted times are $t=$ (a) 2 , (b) 4 , (c) 6, and (d) 10 . narrower as the interfacial shapes become complicated. We mention that the temporal evolution of interfacial structures for the initial distance $d=\pi / 4$ is similar to the one in Fig. 10, but the merging of two interfaces occurs at an earlier time.

Figure 11 shows the growth rates of (a) bubbles and (b) spikes for the single-layer RMI (black line) with the Atwood number $A$ $=0.2$ and the double-layer RMI (blue, green, and red lines) with the Atwood numbers $A_{1}=A_{2}=-0.2$, where the initial distances $d$ are selected as $d=\pi / 4$ (blue lines), $\pi / 2$ (green lines), and $\pi$ (red lines). The growth rates of $I_{1}$ and $I_{2}$ are depicted with solid and dotted lines, respectively. The bubbles and spikes for these Atwood numbers and the linear amplitudes $\left(a_{1}=a_{2}=1\right)$ appear at the same $x$ coordinate on the two interfaces $I_{1}$ and $I_{2}$, where the former appear at $x= \pm \pi$ and the latter appear at $x=0$. Since $a_{1}=a_{2}$, the initial sheet strength $\gamma_{1}(e, 0)$ is equal to $\gamma_{2}(e, 0)$ [refer to Eq. (20)]; therefore, the growth rates of the bubble and spike for $I_{1}$ coincide with those for $I_{2}$
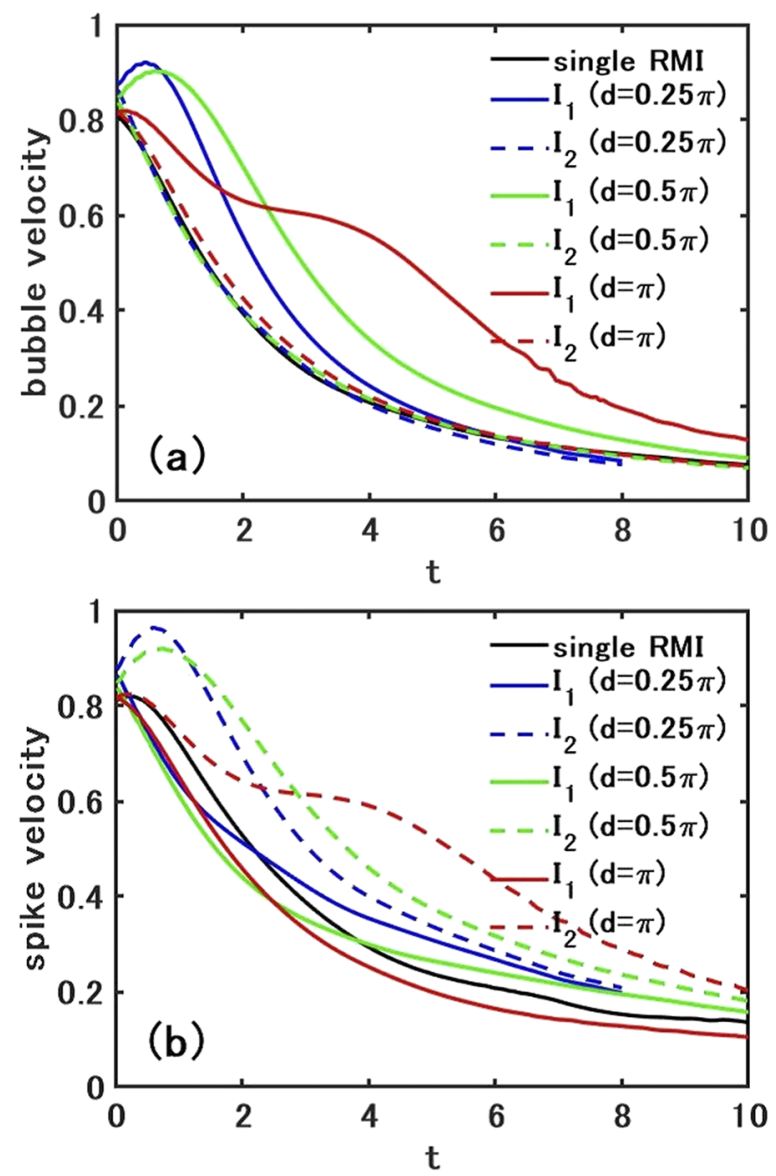

FIG. 11. Temporal evolution of (the absolute value of) the growth rates of (a) bubbles and (b) spikes for $\delta=0.15$, where the Atwood number of the single RM is $A=0.2$ and those of the double-layer are the same as in Fig. 10. The black line denotes the single-layer RMI, and the blue, green, and red lines indicate the growth rates of the double-layer RMI with initial distances $d=\pi / 4, \pi / 2$, and $\pi$. For the double-layer RMI, the solid and dotted lines denote the growth rates of $I_{1}$ and $I_{2}$, respectively. 
at $t=0$. As time passes, the deviation between the sheet strengths $\gamma_{1}$ and $\gamma_{2}$ becomes large due to the nonlinear interaction between the two interfaces, and a difference appears in the growth of bubbles and spikes for $I_{1}$ and $I_{2}$.

The growth rate of the bubble for the single-layer RMI is almost equal to the one for the interface $I_{2}$ (dotted lines) in the double-layer RMI, regardless of the values of the initial distance $d$, and all these lines asymptotically approach zero, the theoretically predicted value for the single-layer RMI. ${ }^{39,40}$ On the other hand, the growth rates of bubbles for $I_{1}$ (solid lines), especially for $d=\pi$ (red solid line), reveal the different behavior from those for the single-layer RMI, although the two lines with $d=\pi / 4$ and $\pi / 2$ asymptotically approach zero. The asymptotic growth rate of the bubble for $I_{1}$ with $d=\pi$ is larger than the one of the spikes [refer to the red solid lines in Figs. 11(a) and 11(b)]. The growth rate of the spike in the single-layer RMI at the earlier stage $(t<1)$ is closest to the one of $I_{2}$ with $d=\pi$ (red dotted line) in the double-layer RMI, and it approaches the line of $I_{1}$ with $d=\pi$ (red solid line) asymptotically. The behavior of the growth rate of bubbles for $I_{1}$ (solid lines with blue, green, and red) is similar to that of spikes for $I_{2}$ (dotted lines with the corresponding colors), although they do not coincide with each other perfectly.

We show the interfacial structures at $t=10$ (the final stage of the calculation) for the double-layer RMI with the initial distance $d=\pi$ (left) and the single-layer RMI for $A=0.2$ (right) in Fig. 12, where the Atwood numbers of the left panel are the same as in Fig. 10. As we see from Fig. 11(b), the asymptotic behavior of the growth rate of the spike in the single-layer RMI (black line) is closest to that of the upper interface $I_{1}$ with the initial distance $d=\pi$ (red solid line), although the interfacial shape, especially the structure in the neighborhood of vortex cores, is quite different from each other, as shown in Fig. 12. Unlike the interfaces with $d=\pi / 2$ in Fig. 10, the bubbles and spikes of the two interfaces with $d=\pi$ keep at a distance even at $t=10$, although the interfaces around the vortex cores merge. Secondary vortex cores with relatively strong sheet strengths appear in the neighborhood of the spike on $I_{2}$ and the bubble on $I_{1}$ at this time in the double-layer RMI.
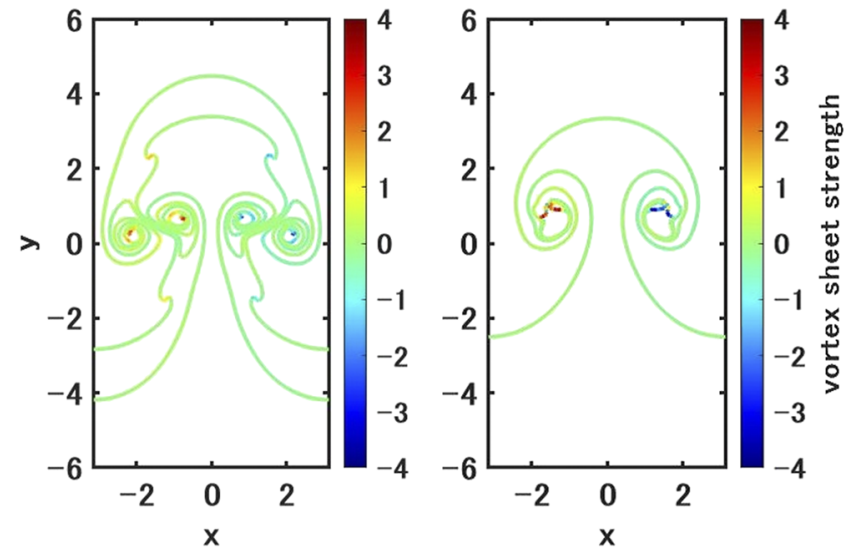

FIG. 12. Comparison between the interfacial structures of the (left) double-layer $\mathrm{RMI}$ and (right) single-layer RMl at the same time $t=10$, where the initial distance in the left panel is $d=\pi$ and the Atwood numbers are the same as in Fig. 10. The Atwood number of the right panel is $A=0.2$.
Now, we compare the interfacial structure with an in-phase initial condition [Eq. (24)] to the one with an out of phase initial condition [Eq. (25)] for fixed Atwood numbers $A_{1}=-0.2$ and $A_{2}$ $=0.2\left[\right.$ case (23b), $\tilde{\rho}_{0}=\tilde{\rho}_{2}=2 / 3$ in (22)] and the initial distance $d$ $=\pi$. Figure 13 shows the interfacial structures (over two periods) at $t=10$ with (a) in phase [Eq. (24), $a_{1}=a_{2}=1$ ] and (b) out of phase [Eq. (25), $a_{1}=1$ and $a_{2}=-1$ ] initial conditions. Since Fig. 13(a) satisfies the in-phase condition (24), the sum of the two initial sheet strengths $\gamma_{1}(e, 0)+\gamma_{2}(e, 0)$ is equal to the initial sheet strength of the single-layer RMI $\gamma(e, 0)$ in the limit of $d \rightarrow 0$ [refer to Eqs. (20) and (21)]; however, unlike the case of Figs. 10-12, the $x$ coordinates
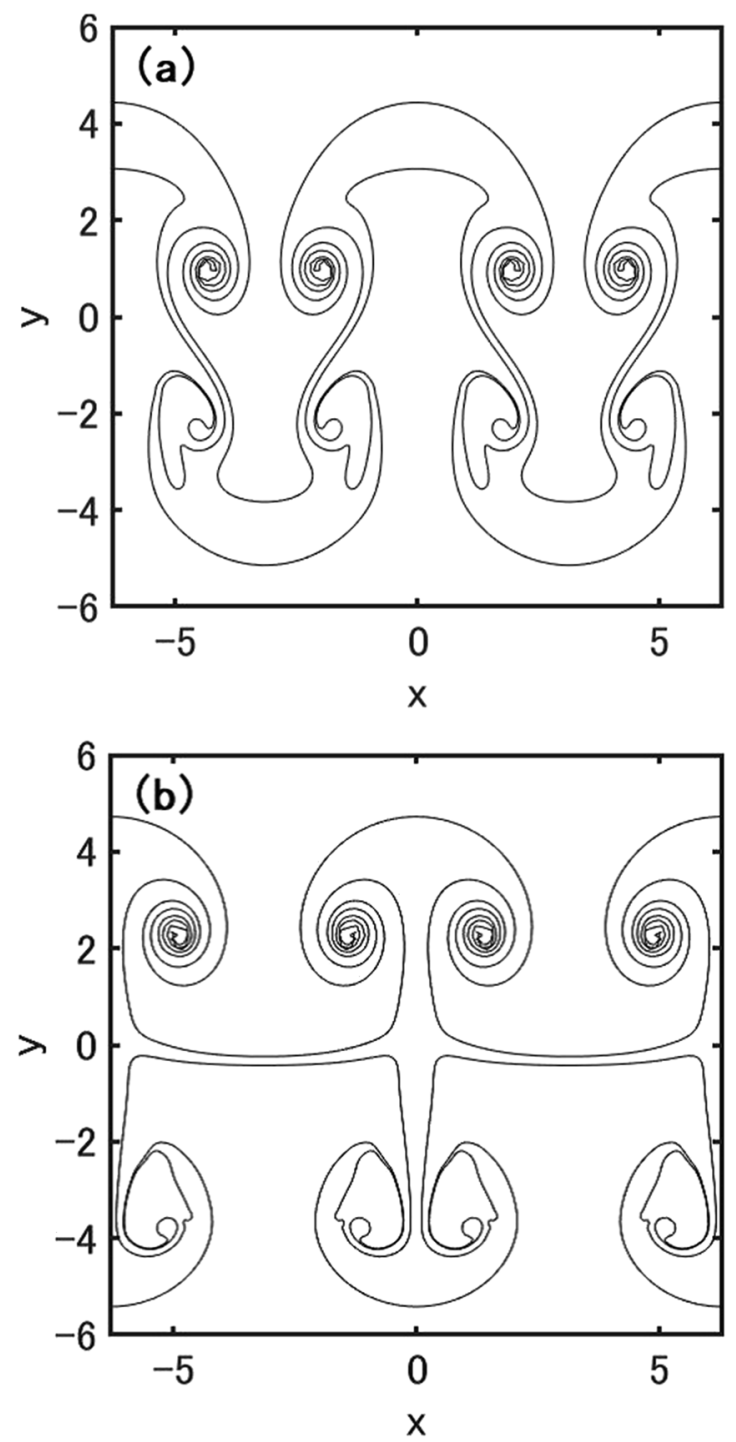

FIG. 13. Interfacial structures at $t=10$ with (a) in phase [Eq. (24), $a_{1}=a_{2}=1$ ] and (b) out of phase [Eq. (25), $a_{1}=1$ and $\left.a_{2}=-1\right]$ initial conditions, where the Atwood numbers $A_{1}=-0.2$ and $A_{2}=0.2$ and the initial distance $d=\pi$. A period of two wavelengths is depicted in these figures. 
of bubbles and spikes are different between $I_{1}$ and $I_{2}$ in this density ratio (the middle layer is the heaviest). Here, the bubble position on $I_{2}$ is $x=0$, and the spike position on $I_{2}$ is $x= \pm \pi$ (in a period) in Fig. 13(a) (the bubble and spike positions on $I_{1}$ are the same as those on $I_{1}$ in Figs. 10-12), while the bubble and spike positions in Fig. 13(b) are $x= \pm \pi$ and $x=0$ (in a period) for both of $I_{1}$ and $I_{2}$. The initial sheet strengths for the out of phase condition [Fig. 13(b)] satisfy $\lim _{d \rightarrow 0}\left[\gamma_{1}(e, 0)+\gamma_{2}(e, 0)\right]=0$ [refer to Eq. (21)]; therefore, this case does not tend to the single-layer solution even in the limit of $d \rightarrow 0$. We see that the curvature of all bubbles tends to almost zero for both figures of Fig. 13, as expected for the conventional singlelayer RMI. ${ }^{39,40}$ The result of Fig. 13(b) with the out of phase initial condition (25) and the density ratios $\tilde{\rho}_{0}<1$ and $\tilde{\rho}_{2}<1$ corresponds to the numerical simulation presented by Zabusky and Zhang. ${ }^{41}$ We mention that the bubble and spike of $I_{1}$ and those of $I_{2}$ are asymmetric with each other unless $A_{1}=A_{2}=0$.
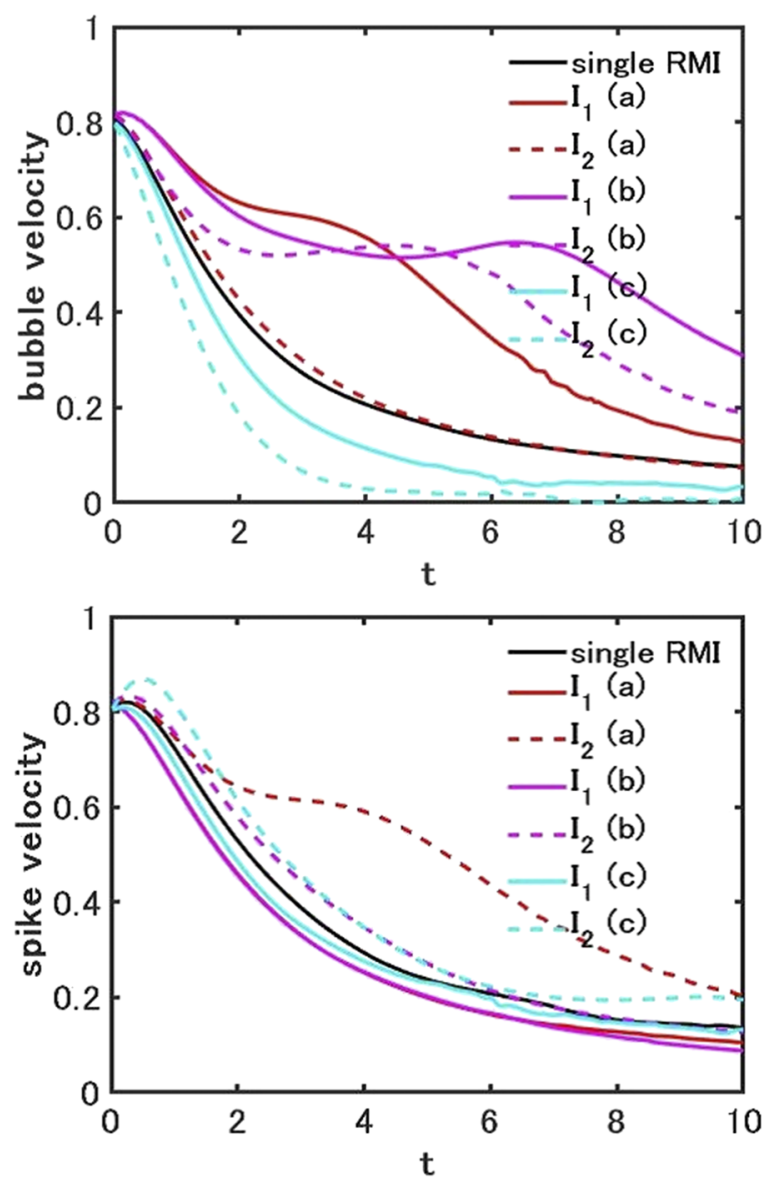

FIG. 14. Growth rates of (upper) bubbles and (lower) spikes for a fixed initial distance $d=\pi$, where the Atwood numbers are (a) $A_{1}=A_{2}=-0.2$ and [(b) and (c)] $A_{1}=-0.2$ and $A_{2}=0.2$ for both panels. The linear amplitudes in (a) and (b) are selected as $a_{1}=a_{2}=1$ (in phase), and those in (c) are $a_{1}=1$ and $a_{2}=-1$ (out of phase). The black solid line denotes the growth rate of the single-layer RMI for $A$ $=0.2$. The growth rates depicted with black and red lines are the same as those in Fig. 11.
The growth rates of bubbles and spikes for two density ratios: case (23a) $\left[\tilde{\rho}_{0}=2 / 3\right.$ and $\tilde{\rho}_{2}=3 / 2, A_{1}=A_{2}=-0.2$ in Eq. (22)] and case (23b) $\left[\tilde{\rho}_{0}=\tilde{\rho}_{2}=2 / 3, A_{1}=-0.2\right.$ and $A_{2}=0.2$ in Eq. (22) $]$ are presented in Fig. 14, fixing the initial distance $d$ as $d=\pi$, where the linear amplitudes in (a) and (b) are selected as $a_{1}=a_{2}=1$ (in phase), and those in (c) are $a_{1}=1$ and $a_{2}=-1$ (out of phase). The asymptotic growth rates of spikes on the upper interface $I_{1}$ are almost the same for (b) and (c) (refer to the pink solid and light blue lines in the lower panel of Fig. 14), which also almost coincide with the growth rates of spikes for $I_{1}$ with $A_{1}=A_{2}=-0.2$ (red solid line in Fig. 14) and the single-layer RMI (black line in Fig. 14). On the other hand, the growth rates of bubbles are all different except the one on $I_{2}$ with $A_{1}=A_{2}=-0.2$ (red dotted line) and the growth rate of the single-layer RMI (black line). In particular, the growth rates of bubbles depicted with pink lines, of which the final interfacial structure is presented in Fig. 13(a), do not tend to zero for both of $I_{1}$ and $I_{2}$.

\section{CONCLUDING REMARKS}

We have investigated the nonlinear interaction of double-layer density stratified interfaces, taking the multi-component RMI as an example. This multi-component RMI is also applicable to the problem of reshock. ${ }^{43}$ We have constructed the governing equations to describe the evolution of the double-layer interfaces using the vortex sheet model (VSM) and performed numerical calculations for both of the regularized parameters $\delta=0$ and $\delta \neq 0$. In the numerical calculations, we obtained the result that the two interfaces approach each other and merge at the final stage of the computation due to the incompressibility. The model equations developed in the current study are easily extended to the $M$-component system $(M \geq 4)$ with $(M-1)$ interfaces with different initial conditions such that the multi-component RTI or multi-layer internal gravity waves if we take the gravity into account, which will be our future work.

We mention that when $A_{1}=A_{2}=0\left(\rho_{0}=\rho_{1}=\rho_{2}\right)$, the bubbles and spikes on $I_{1}$ and $I_{2}$ are perfectly symmetric, and the difference between them as observed in Figs. 10, 12, and 13 is not found, although the merging of two interfaces occurs at the later stage of the evolution. We also remark that when the initial distance $d$ becomes larger (equal to or greater than the wavelength of the initial disturbance), the interaction between the two interfaces becomes weak, and the merging phenomenon does not occur (although the two interfaces approach to some extent).

\section{ACKNOWLEDGMENTS}

The author would like to thank Professor K. Nishihara for valuable discussions. This work was supported by Grant-in-Aid for Scientific Research (C) (Grant Nos. 17K05371 and 18K03418) from the Japan Society for the Promotion of Science, the Osaka City University (OCU) Strategic Research Grant for top priority researches, and the joint research project of ILE, Osaka University.

\section{DATA AVAILABILITY}

The data that support the findings of this study are available from the corresponding author upon reasonable request. 


\section{REFERENCES}

${ }^{1}$ B. R. Sutherland, Internal Gravity Waves (Cambridge University Press, Cambridge, 2010).

${ }^{2}$ P. L.-F. Liu and X. Wang, "A multi-layer model for nonlinear internal wave propagation in shallow water," J. Fluid Mech. 695, 341-365 (2012).

${ }^{3}$ B. Cushman-Roisin and J.-M. Beckers, Introduction to Geophysical Fluid Dynamics: Physical and Numerical Aspects (Elsevier, Amsterdam, 2011).

${ }^{4} \mathrm{~K}$. O. Mikaelian, "Normal modes and symmetries of the Rayleigh-Taylor instability in stratified fluids," Phys. Rev. Lett. 48, 1365-1368 (1982).

${ }^{5}$ K. O. Mikaelian, "Rayleigh-Taylor instabilities in stratified fluids," Phys. Rev. A 26, 2140-2158 (1982).

${ }^{6} \mathrm{~K}$. O. Mikaelian, "Time evolution of density perturbations in accelerating stratified fluids," Phys. Rev. A 28, 1637-1646 (1983).

${ }^{7}$ K. O. Mikaelian, "Richtmyer-Meshkov instabilities in stratified fluids," Phys. Rev. A 31, 410-419 (1985).

${ }^{8}$ W. Liu, X. Li, C. Yu, Y. Fu, P. Wang, L. Wang, and W. Ye, "Theoretical study on finite-thickness effect on harmonics in Richtmyer-Meshkov instability for arbitrary Atwood numbers," Phys. Plasmas 25, 122103 (2018).

${ }^{9} \mathrm{~K}$. Kamemoto, "Formation and interaction of two parallel vortex streets," Bull. JSME 19, 283-290 (1976).

${ }^{10} \mathrm{H}$. G. Lee and J. Kim, "Two-dimensional Kelvin-Helmholtz instabilities of multi-component fluids," Eur. J. Mech.: B/Fluids 49, 77-88 (2015).

${ }^{11}$ R. D. Richtmyer, "Taylor instability in shock acceleration of compressible fluids," Commun. Pure Appl. Math. 13, 297-319 (1960).

${ }^{12}$ E. E. Meshkov, "Instability of the interface of two gases accelerated by a shock wave," Sov. Fluid Dyn. 4, 101-108 (1969).

${ }^{13}$ S. I. Abarzhi, A. K. Bhowmick, A. Naveh, A. Pandian, N. C. Swisher, R. F. Stellingwerf, and W. D. Arnett, "Supernova, nuclear synthesis, fluid instabilities, and interfacial mixing," Proc. Natl. Acad. Sci. U. S. A. 116, 18184-18192 (2019).

${ }^{14}$ T. Inoue, R. Yamazaki, and S.-i. Inutsuka, "Turbulence and magnetic field amplification in supernova remnants: Interactions between a strong shock wave and multiphase interstellar medium," Astrophys. J. 695, 825 (2009).

${ }^{15}$ T. Sano, K. Nishihara, C. Matsuoka, and T. Inoue, "Magnetic field amplification associated with the Richtmyer-Meshkov instability," Astrophys. J. 758, 126 (2012).

${ }^{16}$ T. Sano, T. Inoue, and K. Nishihara, "Critical magnetic field strength for suppression of the Richtmyer-Meshkov instability in plasmas," Phys. Rev. Lett. 111, 205001 (2013).

${ }^{17}$ C. Matsuoka, K. Nishihara, and T. Sano, "Nonlinear dynamics of non-uniform current-vortex sheets in magnetohydrodynamic flows," J. Nonlinear Sci. 27, 531572 (2017).

${ }^{18}$ G. Dimonte, C. E. Frerking, M. Schneider, and B. Remington, "RichtmyerMeshkov instability with strong radiatively driven shocks," Phys. Plasmas 3, 614-630 (1996).

${ }^{19} \mathrm{~J}$. Lindl, Inertia Confinement Fusion: The Quest for Ignition and High Gain Using Indirect Drive (Springer and AIP, New York, 1997).

${ }^{20}$ R. L. Holmes, G. Dimonte, B. Fryxell, M. L. Gittings, J. W. Grove, M. Schneider, D. H. Sharp, A. L. Velikovich, R. P. Weaver, and Q. Zhang, "Richtmyer-Meshkov instability growth: Experiment, simulation and theory," J. Fluid Mech. 389, 55-79 (1999).

${ }^{21}$ K. Nishihara, J. G. Wouchuk, C. Matsuoka, R. Ishizaki, and V. V. Zhakhovsky, "Richtmyer-Meshkov instability: Theory of linear and nonlinear evolution," Philos. Trans. R. Soc., A 368, 1769-1807 (2010).

${ }^{22}$ Y. Zhou, "Rayleigh-Taylor and Richtmyer-Meshkov instability induced flow, turbulence, and mixing. I," Phys. Rep. 720-722, 1-136 (2017).

${ }^{23} \mathrm{~K}$. O. Mikaelian, "Freeze-out and the effect of compressibility in the RichtmyerMeshkov instability," Phys. Fluids 6, 356-368 (1994).

${ }^{24} \mathrm{~J}$. G. Wouchuk and K. Nishihara, "Linear perturbation growth at a shocked interface," Phys. Plasmas 3, 3761-3776 (1996).

${ }^{25} \mathrm{~J}$. G. Wouchuk and K. Nishihara, "Asymptotic growth in the linear RichtmyerMeshkov instability," Phys. Plasmas 4, 1028-1038 (1997).

${ }^{26} \mathrm{~J}$. G. Wouchuk, "Growth rate of the linear Richtmyer-Meshkov instability when a shock is reflected," Phys. Rev. E 63, 056303 (2001).
${ }^{27}$ J. G. Wouchuk, "Growth rate of the Richtmyer-Meshkov instability when a rarefaction is reflected," Phys. Plasmas 8, 2890-2907 (2001).

${ }^{28}$ J. G. Wouchuk, C. H. R. de Lira, and A. L. Velikovich, "Analytical linear theory for the interaction of a planar shock wave with an isotropic turbulent vorticity field," Phys. Rev. E 79, 066315 (2009).

${ }^{29}$ F. Cobos-Campos and J. G. Wouchuk, "Analytical scalings of the linear Richtmyer-Meshkov instability when a shock is reflected," Phys. Rev. E 93, 053111 (2016).

${ }^{30}$ F. Cobos-Campos and J. G. Wouchuk, "Analytical scalings of the linear Richtmyer-Meshkov instability when a rarefaction is reflected," Phys. Rev. E 96, 013102 (2017).

${ }^{31}$ F. Cobos-Campos and J. G. Wouchuk, "Analytic solution for the zero-order postshock profiles when an incident planar shock hits a planar contact surface," Phys. Rev. E 100, 033107 (2019).

${ }^{32}$ C. Matsuoka, K. Nishihara, and Y. Fukuda, "Nonlinear evolution of an interface in the Richtmyer-Meshkov instability," Phys. Rev. E 67(68), 036301 (2003).

${ }^{33} \mathrm{~J}$. W. Jacobs and J. M. Sheeley, "Experimental study of incompressible Richtmyer-Meshkov instability," Phys. Fluids 8, 405-415 (1996).

${ }^{34} \mathrm{C}$. Matsuoka and K. Nishihara, "Vortex core dynamics and singularity formations in incompressible Richtmyer-Meshkov instability," Phys. Rev. E 73, 026304 (2006).

${ }^{35}$ C. Matsuoka and K. Nishihara, "Fully nonlinear evolution of a cylindrical vortex sheet in incompressible Richtmyer-Meshkov instability," Phys. Rev. E 73, 055304(R) (2006).

${ }^{36}$ C. Matsuoka and K. Nishihara, "Analytical and numerical study on a vortex sheet in incompressible Richtmyer-Meshkov instability in cylindrical geometry," Phys. Rev. E 74, 066303 (2006).

${ }^{37}$ M. Vandenboomgaerde, S. Gauthier, and C. Mügler, "Nonlinear regime of a multimode Richtmyer-Meshkov instability: A simplified perturbation theory," Phys. Fluids 14, 1111-1122 (2002).

${ }^{38}$ C. Matsuoka, "Renormalization group approach to interfacial motion in incompressible Richtmyer-Meshkov instability," Phys. Rev. E 82, 036320 (2010).

${ }^{39}$ S. I. Abarzhi, "A new type of the evolution of the bubble front in the RichtmyerMeshkov instability," Phys. Lett. A 294, 95-100 (2002).

${ }^{40} \mathrm{~S}$. I. Abarzhi, K. Nishihara, and J. Glimm, "Rayleigh-Taylor and RichtmyerMeshkov instabilities for fluids with a finite density ratio," Phys. Lett. A 317, 470476 (2002).

${ }^{41}$ N. J. Zabusky and S. Zhang, "Shock-planar curtain interactions in two dimensions: Emergence of vortex double layers, vortex projectiles, and decaying stratified turbulence," Phys. Fluids 14, 419-422 (2002).

${ }^{42} \mathrm{P}$. Colella and P. R. Woodward, "The piecewise parabolic method (ppm) for gas-dynamical simulations," J. Comput. Phys. 54, 174-201 (1984).

${ }^{43}$ G. Malamud, E. Leinov, O. Sadot, Y. Elbaz, G. Ben-Dor, and D. Shvarts, "Reshocked Richtmyer-Meshkov instability: Numerical study and modeling of random multi-mode experiments," Phys. Fluids 26, 084107 (2014).

${ }^{44}$ M. H. Aliabadi and P. Wen, Boundary Element Methods in Engineering and Sciences (World Scientific, 2011).

${ }^{45}$ G. R. Baker, D. I. Meiron, and S. A. Orszag, "Generalized vortex methods for free-surface flow problems,” J. Fluid Mech. 123, 477-501 (1982).

${ }^{46}$ M. J. Shelley, "A study of singularity formation in vortex-sheet motion by a spectrally accurate vortex method," J. Fluid Mech. 244, 493-526 (1992).

${ }^{47}$ T. Y. Hou, J. S. Lowengrub, and M. J. Shelley, "Boundary integral methods for multicomponent fluids and multiphase materials," J. Comput. Phys. 169, 302-362 (2001).

${ }^{48}$ A. J. Chorin and P. S. Bernard, "Discretization of a vortex sheet, with an example of roll-up,” J. Comput. Phys. 13, 423-429 (1973).

${ }^{49}$ R. Krasny, "Computation of vortex sheet roll-up in the Trefftz plane," J. Fluid Mech. 184, 123-155 (1987).

${ }^{50}$ G.-H. Cottet and P. D. Koumoutsakos, Vortex Methods: Theory and Practice (Cambridge University Press, Cambridge, 2000).

${ }^{51}$ G. Baker and J. Beale, "Vortex blob methods applied to interfacial motion," J. Comput. Phys. 196, 233-258 (2004).

${ }^{52}$ G. R. Baker and L. D. Pham, "A comparison of blob methods for vortex sheet roll-up," J. Fluid Mech. 547, 297-316 (2006). 
${ }^{53}$ G. Birkhoff, "Helmholtz and Taylor instability," in Proceedings of Symposia in Applied Mathematics (American Mathematical Society, 1962), Vol. 13, pp. 55-76. ${ }^{54} \mathrm{~N}$. Rott, "Diffraction of a weak shock with vortex generation," J. Fluid Mech. 1, 111-128 (1956).

${ }^{55}$ P. G. Saffman, Vortex Dynamics (Cambridge University Press, Cambridge, 1992).

${ }^{56}$ D. D. Holm, M. Nitsche, and V. Putkaradze, "Euler-alpha and vortex blob regularization of vortex filament and vortex sheet motion," J. Fluid Mech. 555, 149-176 (2006).

${ }^{57} \mathrm{R}$. Krasny, "A study of singularity formation in a vortex sheet by the pointvortex approximation," J. Fluid Mech. 167, 65-93 (1986).

${ }^{58} \mathrm{C}$. Matsuoka and K. Nishihara, "Nonlinear interaction between bulk point vortices and an unstable interface with nonuniform velocity shear such as RichtmyerMeshkov instability," Phys. Plasmas 27, 052305 (2020).

${ }^{59} \mathrm{C}$. Matsuoka, K. Nishihara, and F. Cobos-Campos, "Linear and nonlinear interactions between an interface and bulk vortices in Richtmyer-Meshkov instability," Phys. Plasmas (to be published).

${ }^{60} \mathrm{~A}$. Sidi and M. Israeli, "Quadrature methods for periodic singular and weakly singular Fredholm integral equations," J. Sci. Comput. 3, 201-231 (1998).

${ }^{61}$ R. M. Ker, "Simulation of Rayleigh-Taylor flows using vortex blobs," J. Comput. Phys. 76, 48-84 (1988).

${ }^{62}$ T. Y. Hou, J. S. Lowengrub, and M. J. Shelley, "Removing the stiffness from interfacial flows with surface tension,” J. Comput. Phys. 114, 312-338 (1994).
${ }^{63}$ T. Y. Hou, J. S. Lowengrub, and M. J. Shelley, “The long-time motion of vortex sheets with surface tension," Phys. Fluids 9, 1933-1954 (1997).

${ }^{64}$ D. I. Pullin, "Numerical studies of surface-tension effects in nonlinear Kelvin-Helmholtz and Rayleigh-Taylor instability," J. Fluid Mech. 119, 507-532 (1982).

${ }^{65} \mathrm{~J}$. T. Beale, T. Y. Hou, and J. Lowengrub, "Convergence of a boundary integral method for water waves," SIAM J. Numer. Anal. 33, 1797-1843 (1996).

${ }^{66} \mathrm{R}$. Peyret, Spectral Methods for Incompressible Viscous Flow (Springer, New York, 2002).

${ }^{67}$ G. Baker and A. Nachbin, "Stable methods for vortex sheet motion in the presence of surface tension," SIAM J. Sci. Comput. 19, 1737-1766 (1998).

${ }^{68}$ S.-I. Sohn, D. Yoon, and W. Hwang, "Long-time simulations of the KelvinHelmholtz instability using an adaptive vortex method," Phys. Rev. E 82, 046711 (2010).

${ }^{69}$ M. Herrmann, P. Moin, and S. I. Abarzhi, "Nonlinear evolution of the Richtmyer-Meshkov instability," J. Fluid Mech. 612, 311-338 (2008).

${ }^{70} \mathrm{D}$. W. Moore, "The spontaneous appearance of a singularity in the shape of an evolving vortex sheet," Proc. R. Soc. A 365, 105-119 (1979).

${ }^{71}$ G. Baker, R. E. Caflisch, and M. Siegel, "Singularity formation during RayleighTaylor instability," J. Fluid Mech. 252, 51-78 (1993).

${ }^{72}$ N. J. Zabusky, "Vortex paradigm for accelerated inhomogeneous flows: Visiometrics for the Rayleigh-Taylor and Richtmyer-Meshkov environments," Annu. Rev. Fluid Mech. 31, 495-536 (1999). 